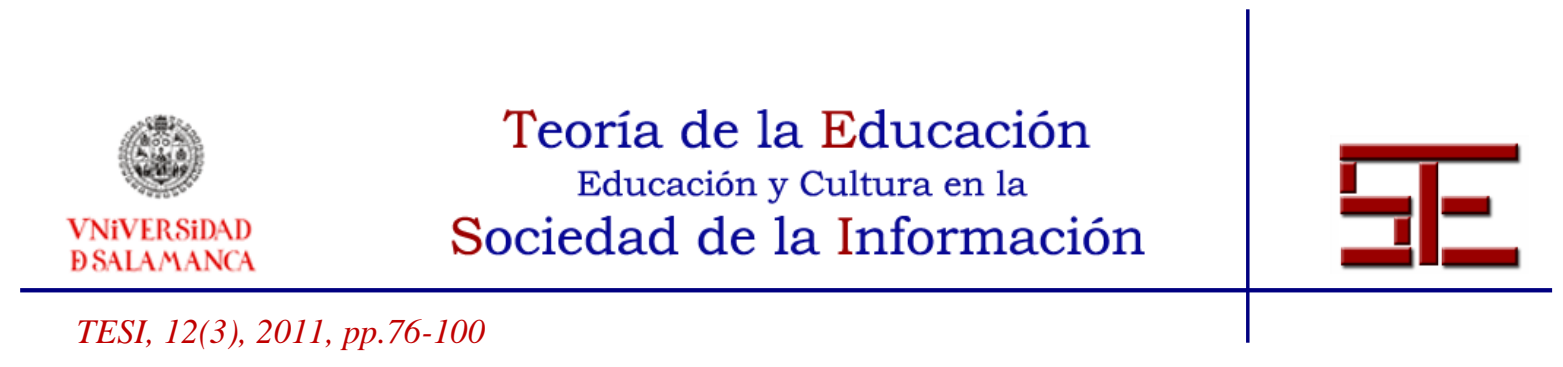

\title{
LA ANALOGÍA Y LA CONSTRUCCIÓN DE SIGNIFICADOS CIENTÍFICOS EN LA ENSEÑANZA DE LA FÍSICA PARA ESTUDIANTES DE INGENIERÍA
}

Resumen: Una analogía puede interpretarse como un mecanismo estructurado de mapeo entre dos dominios, uno llamado dominio-fuente y otro llamado dominio-meta. Esta es una de las funciones que puede cumplir una analogía, y esta función nos interesa en este trabajo para analizar de qué manera específica una analogía participa en la construcción y justificación de conocimientos nuevos. La investigación da cuenta acerca del uso que sobre las analogías como recurso didáctico hacen tres profesoras experimentadas en clases de física universitaria. Más concretamente sobre el papel de tal recurso en la construcción del conocimiento científico y su contribución en el proceso de enseñanzaaprendizaje de la física de nivel universitario y de la formación general del estudiante de ingeniería. El contexto del estudio es la Facultad de Ingeniería de la Universidad de Carabobo, Venezuela. La aproximación metodológica es cualitativa. Es un estudio descriptivo-interpretativo de casos. Los análisis y resultados permiten identificar y tipificar las analogías según el uso dado por las docentes y su contribución en la construcción de significados científicos. Se extraen implicaciones para la mejora de la práctica docente.

Palabras clave: Analogía; Semiótica; Analogía; Enseñanza de la Física.

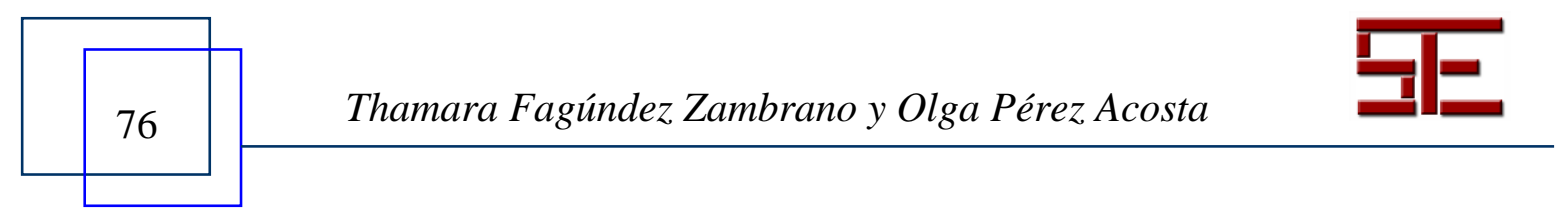




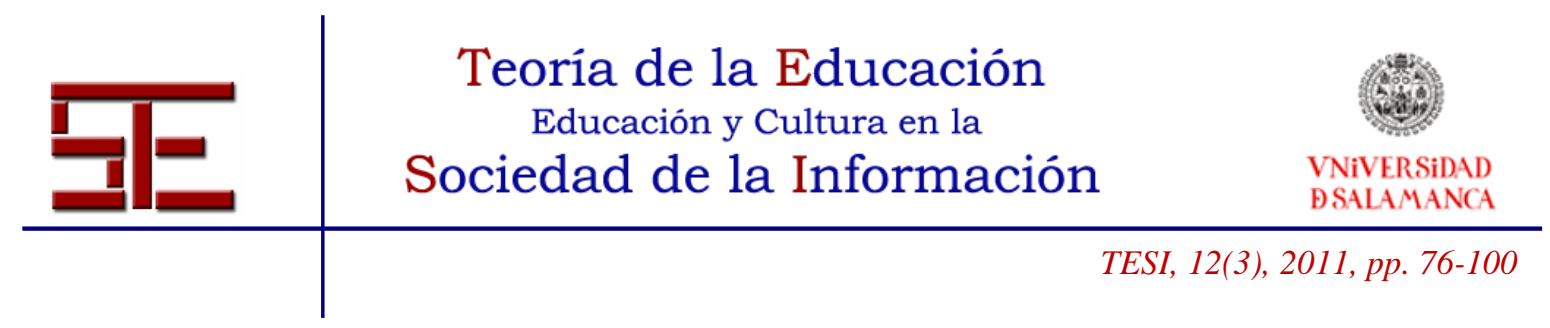

\section{ANALOGY AND CONSTRUCTION OF MEANING IN SCIENCE TEACHING PHYSICS FOR ENGINEERING STUDENTS.}

Abstract: An analogy can be interpreted as a structured mapping between two domains, one called domain-domain and another named source-goal. This is one of functions can play an analogy, and this function we are interested in this work to analyze how specific an analogy involved in construction and justification of new knowledge. The research finds about the use of analogies as a teaching resource are three teachers experienced in college physics classes. More specifically on the role of that resource in the construction of scientific knowledge and its contribution the teaching and learning of college-level physics and general training of the engineering student. The context of the study is Faculty of Engineering, University of Carabobo, Venezuela. The approach methodology is qualitative. This is a descriptive-interpretative cases study. Analysis and results to identify and classify analogies by use given by teachers and their contribution to the construction of meaning scientists. Implications for improving teaching practice are extracted.

Keywords: Analogy; Semiotic; Analogy; Teaching Physics

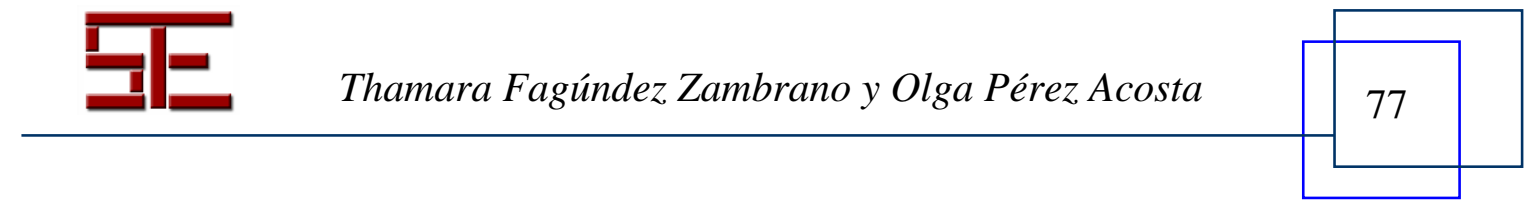




\begin{tabular}{c} 
Teoria de la Educación \\
Educación y Cultura en la \\
$\begin{array}{c}\text { VNiVERSIDAD } \\
\text { BSALAMANA }\end{array}$ \\
\hline TESI, 12(3), 2011, pp.76-100
\end{tabular}

\section{LA ANALOGÍA Y LA CONSTRUCCIÓN DE SIGNIFICADOS CIENTÍFICOS EN LA ENSEÑANZA DE LA FÍSICA PARA ESTUDIANTES DE INGENIERÍA}

Fecha de recepción: 20/08/2011; fecha de aceptación: 22/10/2011; fecha de publicación: 30/11/2011

Thamara Fagúndez Zambrano

tfagunde@uc.edu.ve

Universidad de Carabobo-Venezuela

Olga Pérez Acosta

operez@uc.edu.ve

Universidad de Carabobo-Venezuela

\section{1.- INTRODUCCIÓN}

Independientemente de la especialidad, un aspecto característico de la práctica profesional del ingeniero es su capacidad para resolver situaciones problemáticas. Tal profesional dentro de una organización puede enfrentar problemas relacionados con el control de la producción, análisis de la calidad, mercadeo del producto, compra de materia prima, seguridad e higiene industrial, manejo de inventario y/o almacenes, construcción, electricidad, maquinarias, manejo de líquidos, gases y sólidos, organización, salarios e incentivos al personal, y de otras tantas tareas, responsabilidades o inconvenientes que se presenten día a día. Este trabajo lo convierte en alguien que resuelve problemas mientras mejora el proceso productivo.

El desarrollo de las habilidades y capacidades que requiere el ingeniero para el desarrollo de tales tareas se lleva a cabo durante su etapa de formación en una facultad de ingeniería. En tal formación, 'la física' cumple funciones esenciales en dos aspectos, el cognitivo, promoviendo el aprendizaje de conocimientos básicos fundamentales para el estudio de las ciencias de la ingeniería, y el formativo, promoviendo en el estudiante el desarrollo de capacidades esenciales, como la abstracción, organización, análisis, metodología para resolución de situaciones problemáticas y comunicación. Tales aspectos caracterizan o configuran el 'perfil' del ingeniero. El desafío, entonces, de la enseñanza de la física radica en integrar de forma armoniosa la construcción de significados científicos con el desarrollo de aspectos que contribuyan a la formación integral del estudiante de ingeniería, por lo que supone tomar intencionalmente decisiones sobre la forma preferible para enseñar determinados contenidos con el fin de

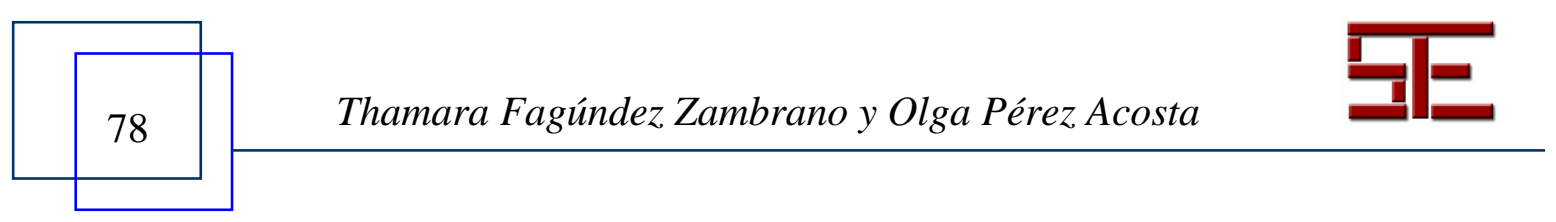




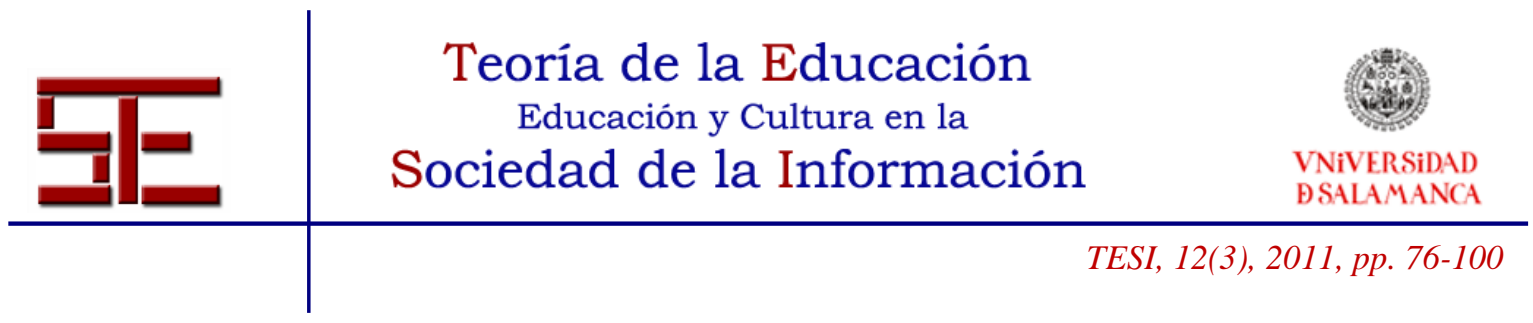

que permitan al alumnado el binomio construcción de significados científicosformación.

Ahora bien, el 'cómo hace' el profesor para presentar a sus alumnos un conocimiento científico adaptado a las necesidades de éstos y al contexto en el que se desempeña, requiere que este conocimiento sea 'transformado o reelaborado' (Chevallard, $1997 \mathrm{y}$ Ogborn, Kress, Martins \& Mc Gillicuddy,1996). Tal reelaboración implica la elección de cómo organizar y presentar los contenidos disciplinares, así como de unos recursos y estrategias didácticas que contribuyan a tal fin. En este aspecto, la analogía se presenta como uno de los recursos usados por los docentes para reelaborar el conocimiento.

El presente estudio se desarrolla con el fin de indagar cómo los profesores utilizan la analogía como recurso en el proceso de enseñanza-aprendizaje de la física de nivel universitario; así como identificar la posible contribución de las mismas en la construcción del conocimiento científico y la formación general del estudiante de ingeniería.

Siendo que las analogías cumplen funciones muy diversas pueden ser consideradas desde perspectivas muy distintas y abarcan aspectos tan diferentes que sólo constituyen un único objeto de estudio a partir de su estructura, nuestro acercamiento al estudio de las mismas se hace desde las perspectivas de la cognición y aprendizaje (Godoy, 2002a), y se orienta a responder las siguientes cuestiones:

a. Desde una perspectiva de la cognición: ¿Cómo se utilizan las analogías para construir explicaciones y argumentaciones?

b. Desde una perspectiva del aprendizaje: ¿Cómo ayudan a comprender mejor un fenómeno o problema? ¿Qué conocimientos son necesarios para sustentar una analogía? ¿Cómo se establecen las relaciones de correspondencia (conexiones) entre la analogía y el concepto? ¿Qué analogías son efectivas y en qué contexto de aprendizaje?

\section{2.- OBJETIVOS DE LA INVESTIGACIÓN}

\section{1.- Objetivo general}

Determinar cómo los profesores utilizan las analogías para construir explicaciones y argumentaciones en el contexto de clases de mecánica universitaria para estudiantes de ingeniería.

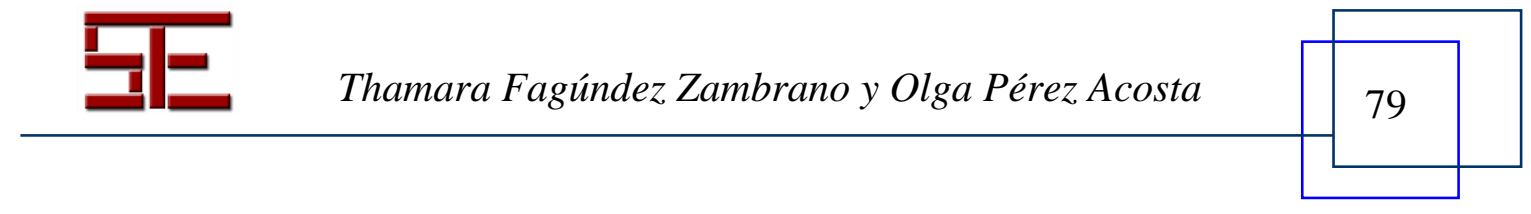




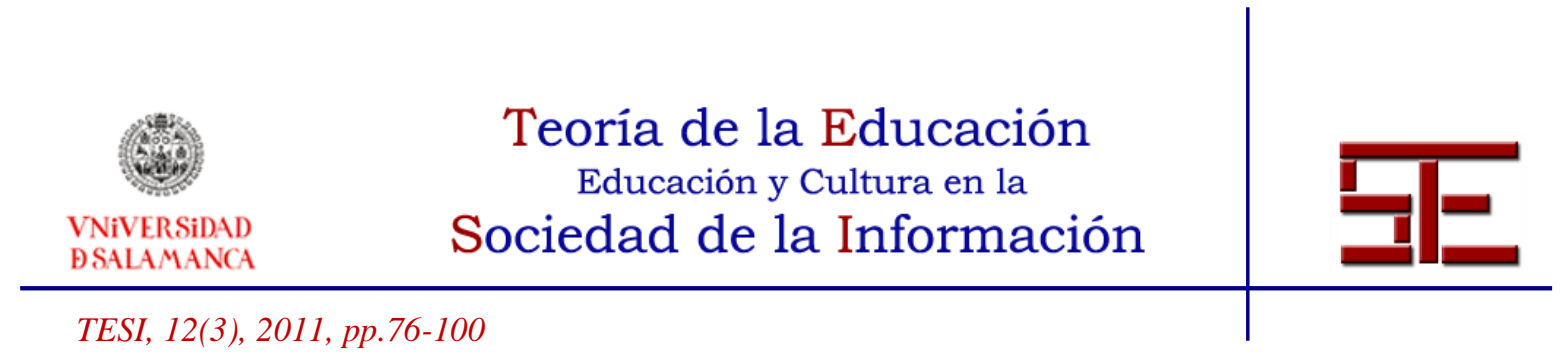

\section{2.- Objetivos específicos}

2.2.1.- Analizar cómo los profesores de física de nivel universitario utilizan las analogías para construir explicaciones y argumentaciones.

2.2.2.- Identificar las analogías presentes en las explicaciones elaboradas en clases universitarias por profesores experimentados de física en el contexto de una facultad de ingeniería.

2.2.3. - Identificar y analizar la contribución de las analogías en las explicaciones para la construcción de significados científicos.

\section{3.- MARCO TEÓRICO}

Una analogía es entendida con frecuencia como una comparación basada en similaridades entre estructuras de dos dominios de conocimiento diferentes, uno conocido y otro desconocido, que mantienen una cierta relación de semejanza entre sí. Constituye una herramienta frecuente en el pensamiento ordinario de las personas y es una forma de razonamiento usada repetidamente en el desarrollo de nuevas ideas en ciencias (Estrada; Godoy, 2007). El objetivo general de su uso es contribuir a comprender una información nueva y, por lo general, se constituyen en una manera de establecer o hacer corresponder los elementos de una nueva idea con los elementos de otra que se encuentra almacenada en la memoria (Lawson, 1993) en forma de 'conocimientos previos o anteriores'.

Desde un punto de vista educativo, sirven para ayudar a comprender una determinada noción o fenómeno, que se denomina objeto, problema o blanco, a través de las relaciones que establece con un sistema análogo (al que también se denomina ancla, base o fuente) y que resulta para el alumno más conocido y familiar (Dagher, 1995a). La analogía es una estrategia educativa utilizada con frecuencia por profesores de ciencias a nivel de secundaria que incorporan analogías en sus explicaciones (Dagher ; Cossman, 1992; Treagust; Duit; Joslin ; Lindauer, 1992; Ogborn; Kress; Martins \& McGillicuddy 1996), y su uso aparece en la literatura fundamentada desde distintos puntos de vista o perspectivas (Oliva; Aragón; Mateo \& Bonat, 2001; Godoy, 2002b). El tipo de aprendizaje por analogía permite la utilización de la estructura de conocimientos previos para extenderla a problemas nuevos.

En el campo de la investigación educativa, el interés en las analogías, entre la diversidad de estrategias didácticas, ha resurgido a partir de la consideración del aprendizaje como proceso de construcción (Oliva, 2004a). Durante algunos años (década de los 80 y principios de la de los 90) se han realizado numerosas investigaciones dirigidas a

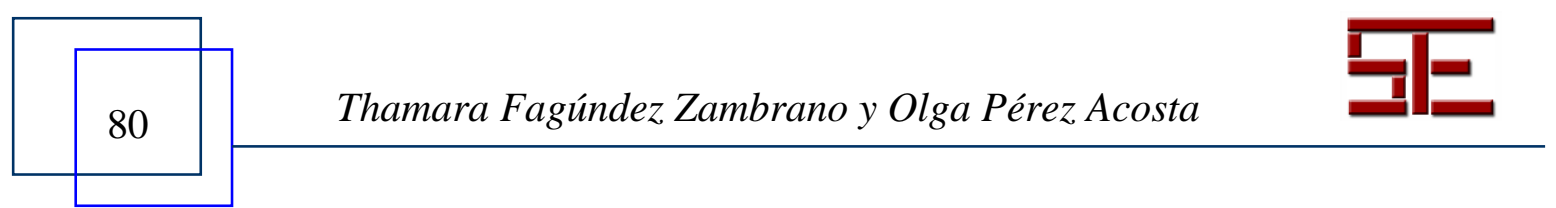




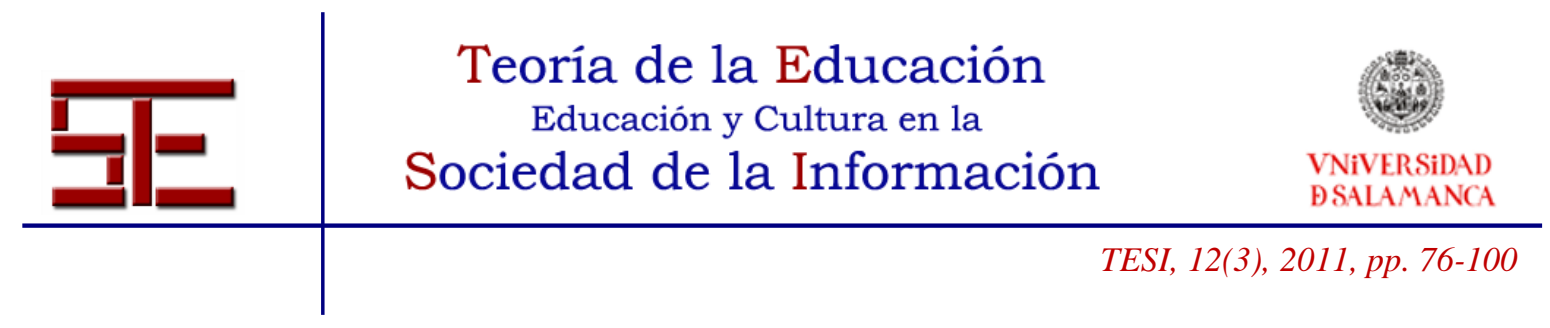

evaluar la efectividad de las analogías como estrategia de enseñanza. Solían aplicarse diseños de tipo experimental o cuasiexperimental que evaluaban los progresos y aprendizajes alcanzados por los alumnos gracias a su empleo. Como mostraba la revisión llevada a cabo por Duit (1991), este tipo de trabajos no condujo a resultados claros e inequívocos al respecto, ya que mientras algunos trabajos apuntaban hacia una influencia positiva de las analogías en el aprendizaje, otros no lograban hacerlo.

En los últimos tiempos, sin embargo, se ha observando un desplazamiento hacia estudios que abordan aspectos relacionados con qué analogías se utilizan, cómo se suelen emplear y qué rasgos didácticos comparten aquellas analogías que parecen obtener cierto éxito con respecto a aquellas que no lo obtienen (Dagher, 1995a). Tales estudios de corte más cualitativo han sido útiles para ofrecer una visión crítica sobre algunas formas de usar las analogías, así como para delimitar algunas dificultades y limitaciones relacionadas con su uso. Pero, sobre todo, han servido para caracterizar cuáles son algunos de los cambios que pueden introducirse para mejorar su validez didáctica (Dagher, 1995a; Oliva et al., 2001).

Las investigaciones que se han realizado sobre analogías abarcan desde su presencia en libros (Fernández; González ; Moreno, 2004); la instrucción analógica (Clement ; Brown, 1984; González, 2005; Oliva, 2006; Oliva y; Aragón 2006; Podolefsky ; Finkelstein, 2006; Zamorano; Gibbs ; Viau, 2006); las concepciones docentes sobre las analogías y su aplicación en el aula (Oliva, 2003; Oliva 2004a), los cambios de concepciones a través de analogías (Clement ; Brown, 1984) hasta las dificultades de su aplicación en el aula (Godoy, 2002a; Moro; Viau; Zamorano ; Gibbs, 2007; Podolefsky ; Finkelstein, 2007a; Podolefsky ; Finkelstein, 2007b).

En relación a las analogías, existen consideraciones de autores respecto a que las mismas permiten potenciar y explicitar el enlace de los conocimientos previos con la información nueva por aprender (Godoy, 2002a; Díaz-Barriga ; Hernández, 2002) y que posibilita la construcción de significados, ya que favorece la visualización de los conceptos científicos que en su mayor parte son abstractos (Godoy, 2007). No obstante, y pesar de lo generalizado del uso de la analogía, su idoneidad como recurso en el aula se ha cuestionado, encontrándose una polarización de opiniones a favor y otras en contra en relación al uso de la misma como recurso didáctico. Tal polarización de opiniones ha originado el desarrollo de un gran número de estudios con el objeto de evaluar su incidencia en la práctica docente. Sobre este tema pueden encontrarse hoy día un gran número de investigaciones. Los resultados obtenidos a lo largo de este conjunto de investigaciones no son concluyentes, como lo muestra Duit (1991). Lo anterior se debe, según Oliva (2003), a que junto a trabajos en los que se obtienen resultados

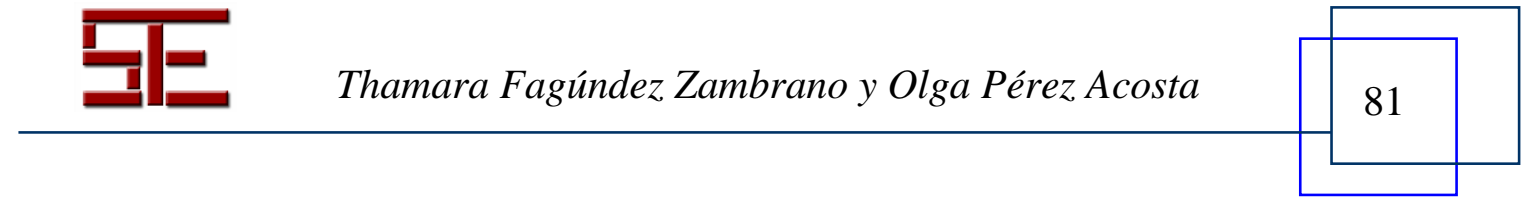




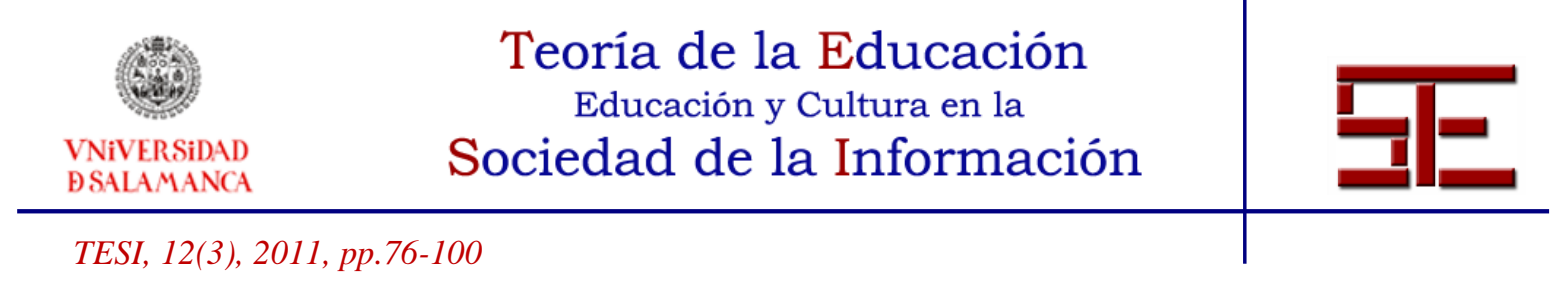

positivos aparecen otros que constatan una aportación más limitada de las mismas; incluso, en algunos casos, se han llegado a detectar ciertas dificultades y peligros de su uso.

Dagher (1995a) realizó una segunda revisión, más profunda, analizando cualitativamente qué rasgos comparten aquellas investigaciones en las que sí parecen tener éxito las analogías con respecto a aquellas otras que no lo obtienen. El resultado de este análisis concluye que el debate no debe centrarse en si las analogías en la enseñanza son o no útiles, sino en las condiciones a partir de las cuales las analogías pueden llegar a resultar útiles desde el punto de vista didáctico, lo que implica esclarecer los rasgos metodológicos que pueden mejorar su efectividad en la enseñanza (Oliva, 2003).

En relación a la enseñanza de la física, no hay evidencia contundente sobre la efectividad de las analogías para tal fin, por lo que se han realizado estudios en los que se concluye que existen factores que influyen en el aprendizaje que se puede obtener empleando esta estrategia (Podolefsky ; Finkelstein, 2007).

\section{4.- METODOLOGÍA DE LA INVESTIGACIÓN}

Se trata de una investigación con una aproximación metodológica cualitativa y estudio instrumental colectivo de casos (Stake, 1998). Los participantes en el estudio son tres profesoras experimentadas de física de la Facultad de Ingeniería de la Universidad de Carabobo, Venezuela. Los datos son fundamentalmente relatos de episodios de clases de física de los primeros cursos de la Facultad de Ingeniería de la Universidad de Carabobo (Venezuela) recogidos a partir de grabaciones en video y notas de campo de las investigadoras que han actuado como observadoras no participantes. Las grabaciones se han transcrito en una plantilla que recoge tanto la parte verbal como la parte multimodal. Las explicaciones de las profesoras se han dividido en episodios en base a ser una unidad de contenido que tiene significado en sí mismo.

\section{5.- ANÁLISIS Y DISCUSIÓN}

En este apartado presentamos el análisis mostrando el estudio de algunos fragmentos de las clases seleccionadas. Las analogías incorporadas permiten dotar de cierto significado inicial a los términos científicos involucrados; a la vez que pueden sugerir nuevas ideas y hacer más comprensibles los significados científicos.

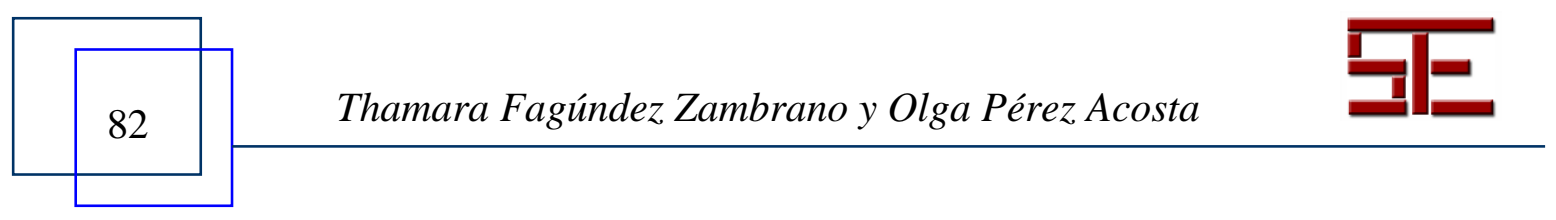




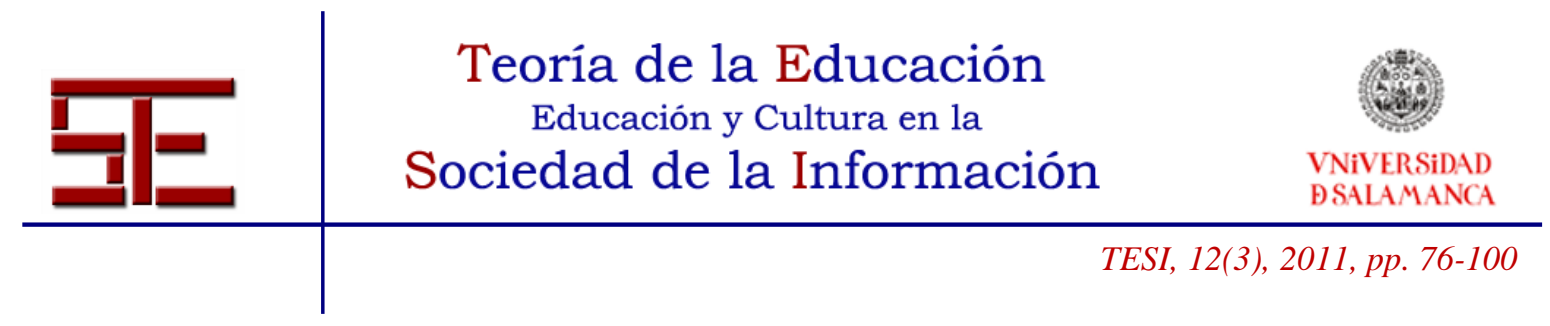

En términos generales en las clases de física analizadas las analogías son usadas para:

1. La construcción de significados relacionados con conceptos científicos abstractos

2. Establecer el comportamiento de entidades y/o sistemas físicos

3. Establecer y/o referenciar un marco teórico de análisis

4. Como referencia para establecer una metodología y/o procedimiento

\subsection{Analogías para la construcción de significados relacionados con conceptos científicos abstractos}

En el segmento explicativo siguiente la profesora A reelabora la entidad 'campo gravitatorio' usando como recurso para su construcción un razonamiento basado en la analogía. En este caso plantea una analogía estableciendo una relación de semejanza entre dos entidades pertenecientes a áreas de conocimiento diferentes; una entidad científica (un campo), desconocida para los alumnos en ese momento; y una entidad no científica (un aura) de un campo diferente, pero quizás más cercano y/o cotidiano a los ojos de los alumnos. Aquí, el interés de la profesora es la construcción de significados acerca de la entidad 'campo', para luego realizar otras tareas orientadas a la elaboración de otra entidad: 'el campo gravitatorio':

\footnotetext{
Profesora: ...Vamos a hablar un poquito acerca del campo gravitatorio. Me gustaría que aprovechemos este tema para hablar de lo que significa un campo. ¿Qué es un campo?

Alumno 1: Un espacio.

Alumno 2: Un espacio alrededor de algo.

Profesora: [Hace gesto facial de inconformidad: G1] [...] Un campo [...] una propiedad cualquiera, la masa, las cargas eléctricas [...] el movimiento de cargas eléctricas producen campos. Tenemos entendido que un campo o lo visualizamos así -y eso es- es algo así como un aura [Hace movimiento con la mano izquierda describiendo un semicírculo para ilustrar la idea de Aura: G2] alrededor de esa propiedad.
}

Observamos que la incorporación de la analogía entre el 'campo' y el 'aura', en la explicación elaborada, no presupone en ningún momento la existencia de una igualdad de forma simétrica entre las mismas; establece una semejanza entre las entidades, con un propósito dado. La analogía remite, como lo hace con frecuencia, a una especie de imagen del concepto cotidiano como referente del concepto científico abstracto que la profesora quiere enseñar. Consideramos que contribuye a la construcción de sentido y significados respecto a la entidad "campo", a partir de los significados asociados a la entidad "aura". Tomando como referencia a Glynn, Britton, Semrud-Clikman, \& Muth, (1989) detectamos que la función de la analogía, en el caso del episodio que

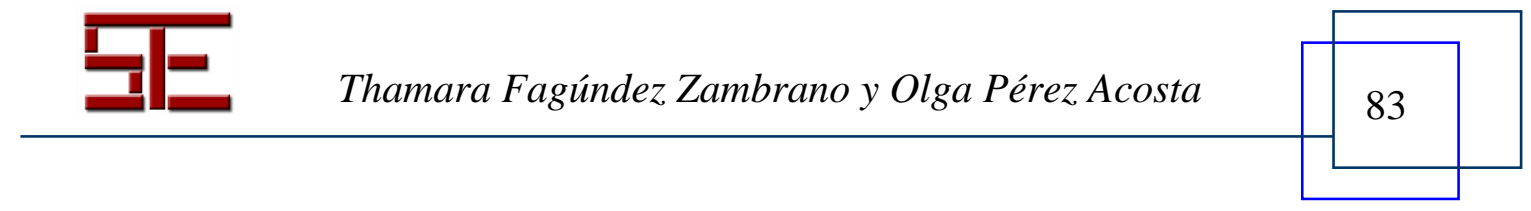




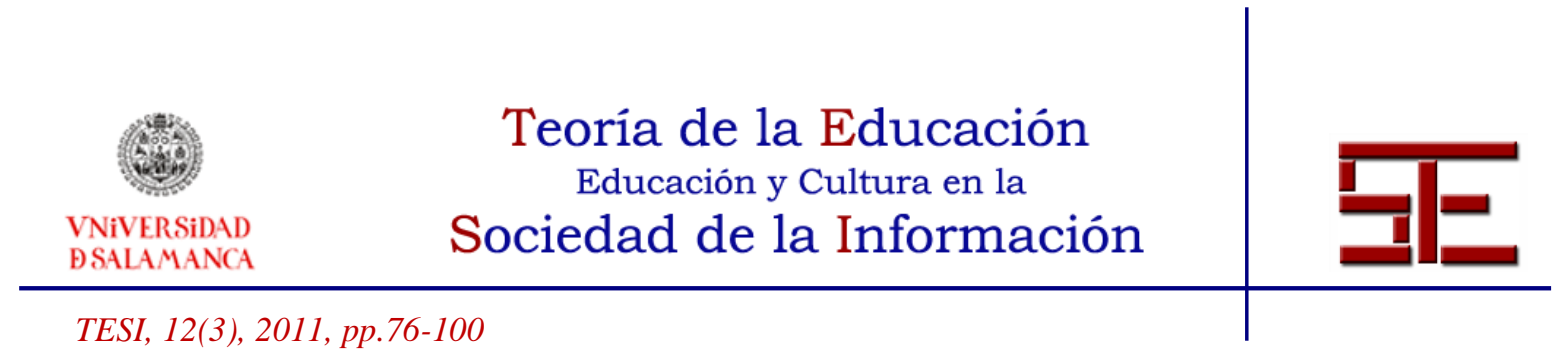

presentamos, es explicativa, por su orientación a 'mediar' entre los dos campos (científico y no científico), con el fin de contribuir a que los alumnos inicien la construcción de la entidad abstracta 'campo' mediante su correspondencia con una entidad más cotidiana.

En otro pasaje explicativo del mismo episodio, observamos a la profesora A intentando elaborar la entidad 'campo gravitatorio'. A grandes rasgos, desde el punto de vista de la física, un campo eléctrico o magnético es la zona en que se manifiestan los efectos de esas fuerzas, un campo de acción. En el mismo episodio, encontramos un pasaje en el que, si bien no observamos la estructura 'tradicional' de una analogía, la interpretamos y percibimos como una 'analogía tácita' incorporada por la profesora A cuando expresa:

"El campo gravitatorio solamente apunta hacia el centro de la Tierra...en el caso de las cargas, el campo puede ser hacia las cargas...cuando sean negativas...y hacia fuera de las cargas cuando estas son positivas..."

Consideramos que en esta frase la profesora A está comparando, y a la vez diferenciando, un aspecto característico del campo gravitatorio: el hecho de que siempre apunta hacia el centro terrestre, con el campo producido por cargas eléctricas; que podrá ser hacia las cargas (cuando sea generado por cargas negativas) o desde las cargas (cuando lo generen cargas positivas). Las analogías anteriores contribuyen a que los alumnos comprendan y visualicen mejor la nueva entidad que se introduce; y que se caracteriza por su carácter abstracto, porque les aportan un marco que la hace más 'familiar' y por tanto próxima a sus experiencias cotidianas y a su conocimiento previo, reduciendo también notablemente las demandas de abstracción.

En otro episodio analizado encontramos cómo la profesora B usa la analogía como recurso para la elaboración de la entidad momento de inercia. Para tal elaboración, la profesora $\mathrm{B}$, una vez que recuerda al grupo clase el aspecto referido a la masa y su conceptualización como "tendencia de los cuerpos a mantener el estado de movimiento o reposo; y que no se modifica a menos que actúen fuerzas externas sobre el”; incorporando adicionalmente la noción de que también se le podría ver como "una medida de la resistencia de un cuerpo a cambiar su estado de movimiento o reposo", inicia la elaboración de una nueva entidad relacionada al movimiento de rotación pura: el 'momento de inercia', mediante una analogía. A continuación el segmento comentado:

Profesora: ...comenzamos hoy con el estudio de las causas del movimiento de rotación pura de los cuerpos [escribe este título en el pizarrón E1]. Así como en la dinámica de traslación nosotros estudiamos las causas que originaban el movimiento de traslación de los cuerpos, vamos

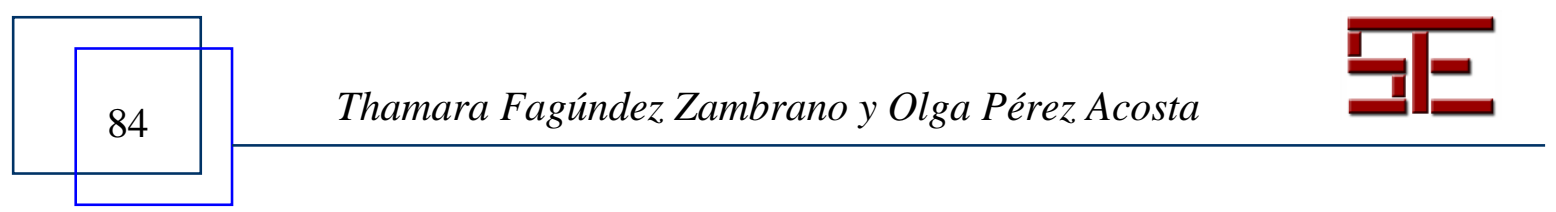




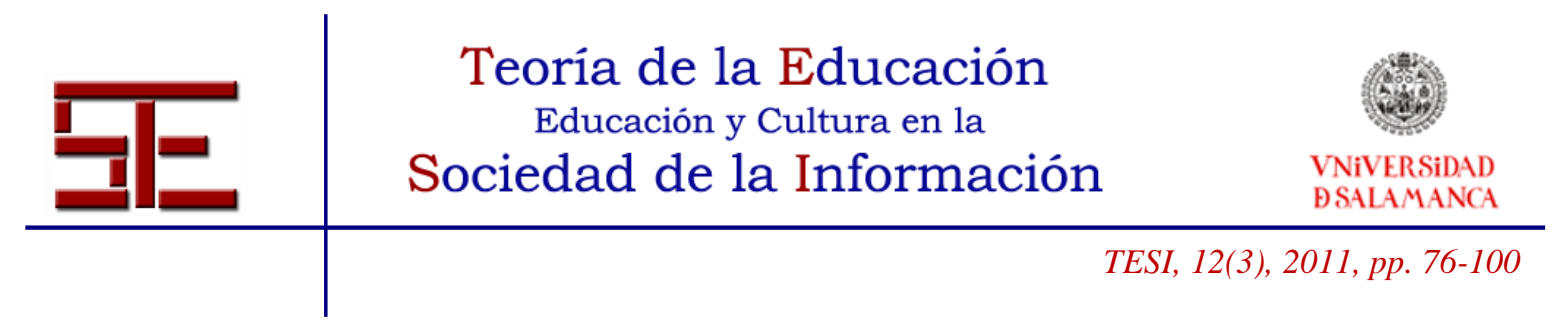

ahora a hablar de las causas que provocan el movimiento de (rotación pura). Vamos a hacer un bosquejo de lo que vamos a estudiar en la dinámica de rotación conocida ya, la dinámica de traslación y después con detalle vamos a hablar de cada una...Vamos entonces a revisar el movimiento de traslación desde el punto de vista dinámico e iremos incorporando los análogos rotacionales correspondientes.

Profesora: Muy bien, en el movimiento de traslación, la masa [escribe nombre y símbolo: E3] podíamos relacionarla con la (inercia), que es la tendencia de los cuerpos a mantener el estado de movimiento o reposo; y que (no) se modifica a menos que actúen fuerza externas sobre el. Podíamos verla como una medida de la (resistencia) de un cuerpo a cambiar su estado de movimiento o reposo. Pues en rotación, (el momento de inercia es a lo que es la rotación lo que la masa es a la traslación). ¿Okey?, ¿captaron la idea? [Mira al grupo] Alumnos: $\mathrm{Si}$

Profesora: ¿Qué es el momento de inercia, entonces?

Alumnos: La resistencia que ofrece un cuerpo a cambiar su reposo o movimiento de rotación pura.

Profesora: [G1]. En el movimiento de rotación, también tendremos, entonces, (una variable) que nos informa, ahora, de la resistencia que ofrece un cuerpo (ahora) a cambiar su estado (de reposo o movimiento de rotación pura). Esta variable se denomina (momento de inercia) [escribe Momento de inercia en la pizarra. E4] y lo representamos con la letra I [escribe I en la pizarra: E4]. Entonces una medida de la resistencia que ofrece un cuerpo a cambiar su estado de reposo o movimiento de rotación pura nos la aporta el momento de inercia. Este momento de inercia se escribe con un subíndice o [lo escribe al lado de la letra I: E4] que nos va a indicar (el eje) en torno al que el cuerpo está girando. Más adelante veremos la expresión que utilizaremos para determinar numéricamente, como siempre, los números son parte de la física y la ingeniería; entonces conoceremos qué expresión nos va a permitir calcular el momento de inercia por ejemplo, de un mismo cuerpo cuando este gira alrededor de ejes diferentes. Veremos que no es lo mismo que este [toma el borrador en la mano izquierda] borrador gire en torno a este eje [acción: hace girar el borrador sujetándolo por uno de sus extremos] que hacerlo girar en torno a este eje [Acción: gira al borrador sujetándolo ahora en su punto medio]. Es decir, la (resistencia) del cuerpo a girar respecto a este o este eje [vuelve a girar el borrador sujetándolo en los dos puntos anteriores] es diferente. Y una medida de esa resistencia es el momento de inercia...

...En el movimiento de rotación, también tendremos, entonces, (una variable) que nos informa, ahora, de la resistencia que ofrece un cuerpo (ahora) a cambiar su estado (de reposo o movimiento de rotación pura). Esta variable se denomina (momento de inercia) [escribe Momento de inercia en la pizarra. E4] y lo representamos con la letra I [escribe I en la pizarra: E4]. Okey, hablamos de [...], ¿de qué más hablamos?, ¿a ver? [mira al grupo y luego a un alumno (alumno 1) y un movimiento de la cabeza le indica a este que espera una respuesta de el].

Alumno1. La fuerza.

Alumno 2: El momento lineal.

Profesora: [Asiente con la cabeza: G1] Del momento lineal y de la fuerza [escribe los nombres y la nomenclatura: E5] en donde la expresión [escribe expresión de la segunda ley y ese nombre: E6] expresa que la fuerza es igual a la variación instantánea del momento lineal que experimenta el cuerpo. En el movimiento de traslación dijimos que el movimiento de las partículas se debía a

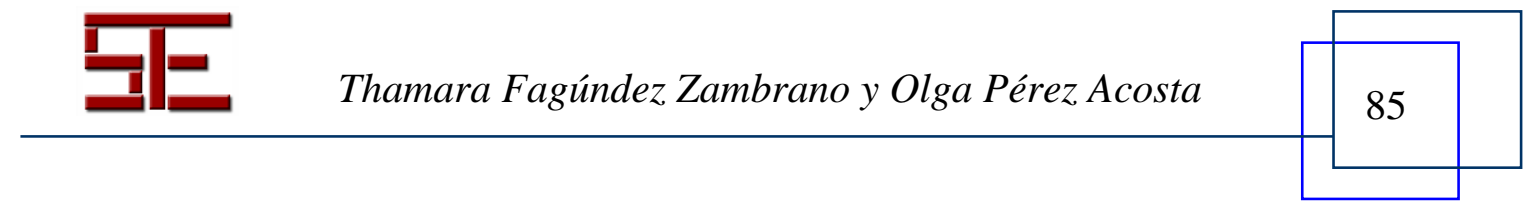




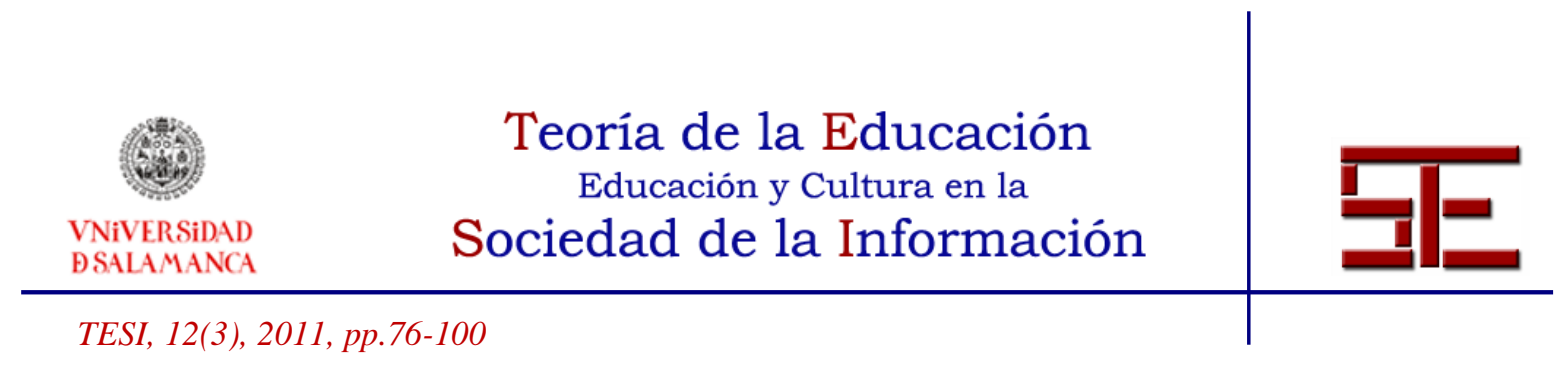

la interacción de las partículas con el medio y que esa interacción la llamábamos fuerza. Bien, en rotación tenemos que la causa del movimiento de rotación se llama (torque o momento de rotación) [escribe eso y el símbolo: E7] y se expresa, al igual que antes en función del (momento angular) [escribe eso y el símbolo: E8] como la variación instantánea de él, del momento angular [escribe eso y el símbolo: E9]...

En este segmento el planeamiento de la analogía sigue la "fórmula general A es a B como C es a D” (Perelman ; Olbrechts-Tyteca, 1989: 570). Es uno de los pocos episodios en los que encontramos la analogía planteada explícitamente de esta forma. En este mismo segmento, y en el que explícitamente la profesora inicia el estudio de dinámica del movimiento de rotación pura, el punto de partida de la explicación se fundamenta en los conocimientos previos del grupo clase respecto al estudio dinámico del movimiento de traslación. En este caso la selección de la profesora se orientó a la elaboración y construcción de significados en relación a las entidades que permiten estudiar las causas del movimiento de rotación pura, estableciendo analogías con las diversas entidades que permiten el estudio de las causas del movimiento de traslación.

Una vez presentada la analogía entre masa y momento de inercia, se limita a establecer otras analogías, pero de forma 'implícita', a la vez que continúa elaborando la lista' de variables que permitirán el estudio del movimiento de rotación pura, repitiendo el mismo procedimiento que describimos antes, para cada una. En la pizarra, el esquema resultante de este proceso fue el que se muestra en la Figura 1:

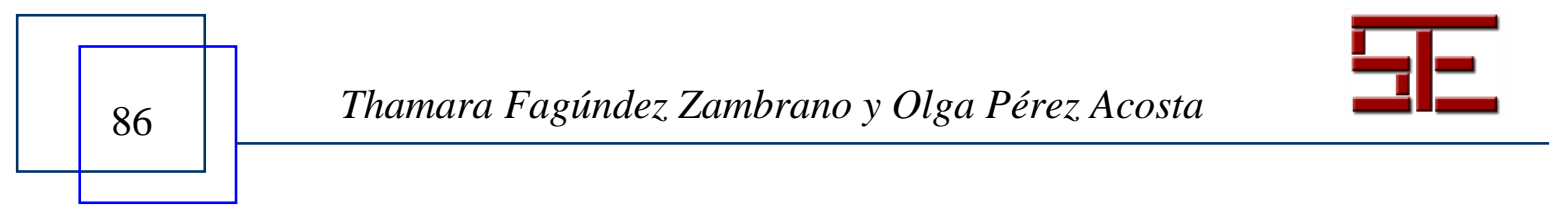




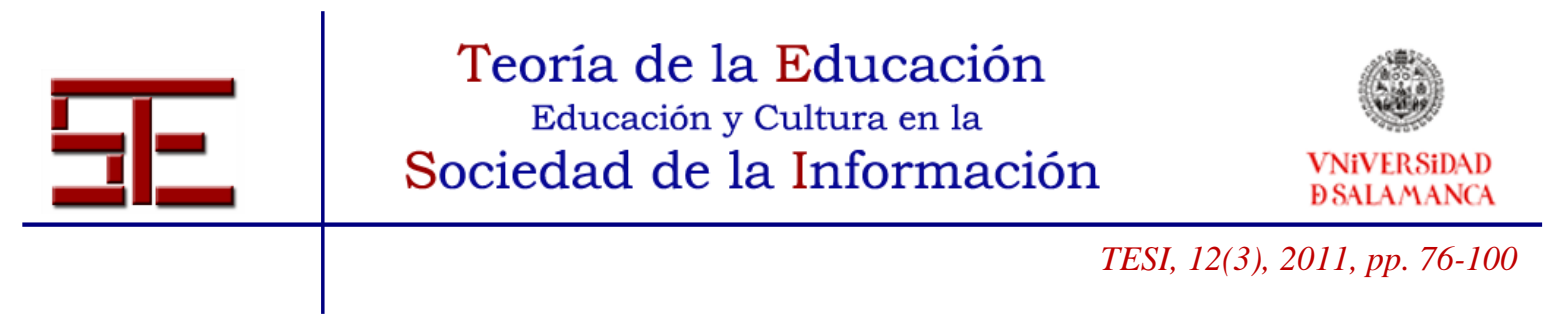

Movimiento de Traslación: Movimiento de Rotación:

Masa $(m)$ (I)

Momento Lineal $(\vec{p})$

Fuerza $(\vec{F}) \quad$ Torque $(\vec{\tau})$

Segunda ley $\left(\vec{F}=\frac{d \vec{p}}{d t}\right)$

Expresión de la segunda ley para Análogo rotacional de la segunda cuerpos de masa constante $(\vec{F}=m \cdot \vec{a} \quad$ ley $(\vec{\tau}=I . \vec{\alpha})$ )

Energía cinética $\left(\mathrm{K}=\frac{1}{2} m v^{2}\right) \quad$ Energía cinética rotacional $\left(K=\frac{1}{2} I \omega^{2}\right)$

Figura 1: Establecimiento de analogías entre variables dinámicas del movimiento de traslación y las del movimiento de rotación.

Observamos que el planteamiento de las 'analogías implícitas' siguientes era compartido y admitido por los alumnos, tomando como criterio las sucesivas interacciones entre alumnos y profesora y que en ningún momento éstos dieron 'muestras' de incomprensión (y que la profesora buscaba captar por medio de preguntas, la mirada y/o de interpretar los silencios de los alumnos). Al final del proceso, las diferentes variables para el estudio dinámico del movimiento de rotación se vieron plasmadas en el pizarrón en un esquema que paulatinamente se fue elaborando a medida que se hacían las analogías.

\subsection{Analogías para establecer el comportamiento de entidades y/o sistemas físicos}

Según la Ley de la Gravitación Universal de Newton la región en que actúan las fuerzas gravitatorias puede considerarse como un campo gravitatorio. Este conocimiento orienta a la profesora A a elegir para la elaboración del 'efecto del campo gravitatorio' el camino de la analogía entre el mismo y el efecto del campo eléctrico. Tal elaboración la vemos en la Tabla 1:

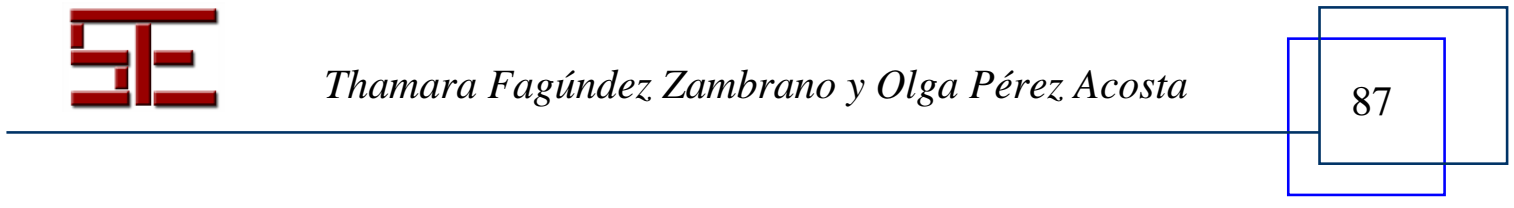




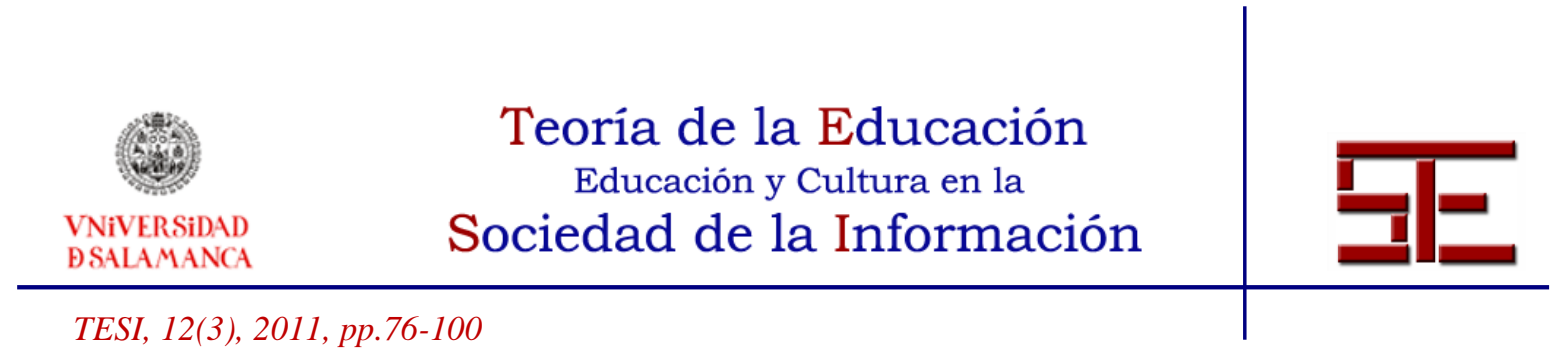

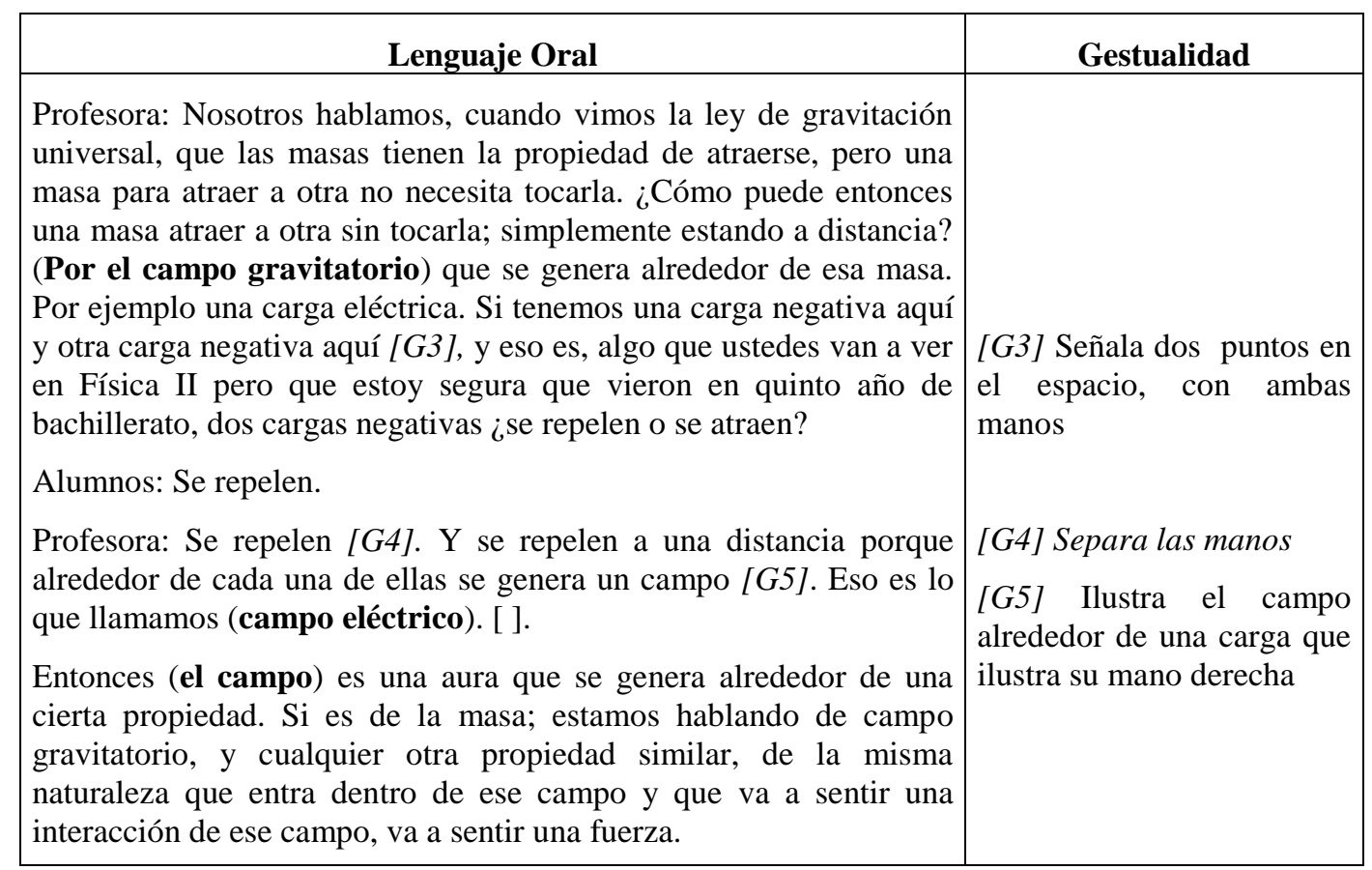

Tabla 1: Elaboración de entidades por la analogía.

Aun cuando en el segmento no visualizamos el establecimiento explícito de una analogía, en el mismo captamos la elección de la profesora de este camino de razonamiento analógico para la construcción de significados. La profesora A ha elaborado la entidad 'efecto del campo gravitatorio' de la forma como lo hace, basándose en el de entidad campo eléctrico, tal vez porque sabe que, en general, los estudiantes de ingeniería tienen interés en esta área, la electrónica y la computación, que está muy basada en conocimientos de electromagnetismo. Además, el currículo de bachillerato contempla prácticas de laboratorio de física donde los alumnos pueden visualizar la atracción o repulsión de cargas de prueba (de cargas positivas) en presencia de campos generados por cargas positivas y negativas. Esto último que comentamos, suponemos que orienta a la profesora A, finalmente, a intentar que los alumnos elaboren significados referidos al efecto del campo gravitatorio tomando, también como referencia, sus conocimientos sobre la entidad 'campo eléctrico' y el efecto de este campo sobre cargas de prueba.

En otro episodio encontramos una situación interesante, por el hecho de que la profesora $\mathrm{C}$, en su intento por construir la entidad fuerza no conservativa, elabora una explicación

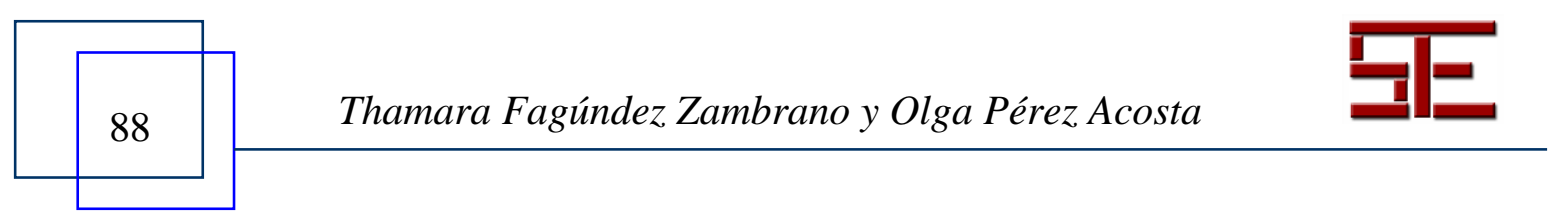




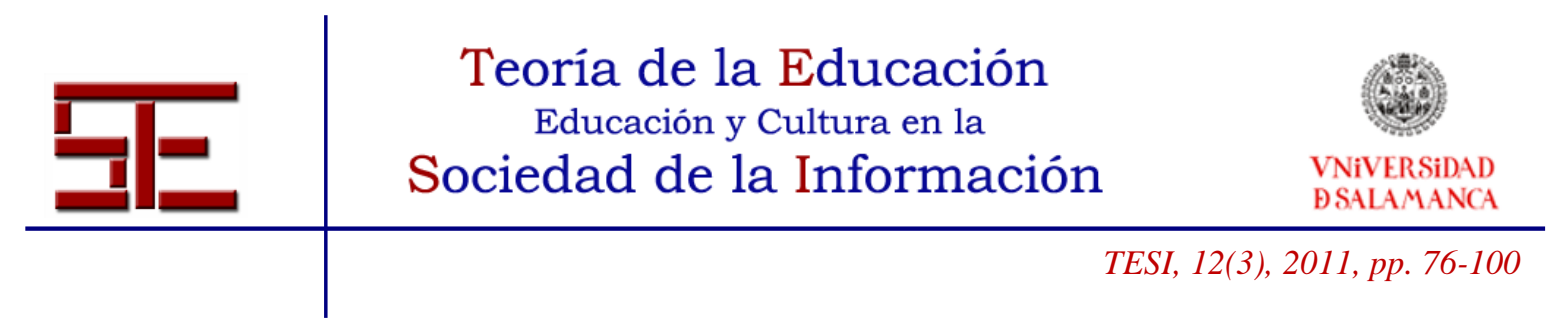

en la que incorpora una doble analogía: una en la que compara el 'efecto del roce en el desgaste de la tiza' con el 'efecto de éste en la energía', y otra en la que compara el 'efecto del roce en la energía' con el 'efecto del peso en la energía'.

Profesora: ...Estamos hablando acerca de que el peso no produce pérdidas de energía; okey, puede que produzca un descenso de energía cinética, pero simultáneamente va aumentando la potencial gravitatoria; entonces podemos decir que no importa; una forma u otra de energía, pero la tenemos; solo existe transformación de un tipo de energía a otra. Cuando hablamos del roce ¿qué pasa? Imagínense ustedes que yo cojo un bloque gigante de tiza y lo llevo arrastrando desde aquí hasta el Decanato; pero cuando llego al decanato me doy cuenta que dejé un camino blanco de aquí hasta allá. Entonces y para que nadie se dé cuenta me regreso arrastrando la tiza exactamente por la misma trayectoria para recoger la tiza que dejé regada. ¿qué va a pasar?

Alumnos: Varias respuestas ininteligibles.

Profesora: Que el camino va a quedar más blanco todavía, porque la masa que perdí en mi viaje de ida no la voy a recuperar en mi regreso (¿por qué?) (Porque el roce produce desgaste), porque lo que el roce hace que se pierda no se puede recuperar; lo mismo pasa con la energía, el efecto del roce en la energía es igual; no es como el efecto del peso; cuando un cuerpo cae desde una altura h, por ejemplo - [Ubica la mano izq. a una altura dada]- sin considerar la resistencia del aire sabemos que en virtud de su posición tiene almacenada una cierta energía potencial (energía potencial gravitatoria); a medida que el cuerpo cae pierde energía potencial -que depende de su posición-; pero gana energía cinética que depende de su movimiento de tal manera que al final la energía potencial que se pierde en el camino se recupera, pero en forma de otra energía (la cinética). La energía se transformó [ ]. El roce o fuerza de roce no, el roce no permite la recuperación de la energía que se queda en el camino; por eso el roce es una fuerza [ $i$ ?].

Editando el discurso de este segmento tenemos: "El peso no produce pérdidas de energía... puede que produzca un descenso de energía cinética, pero simultáneamente va aumentando la potencial gravitatoria... solo existe transformación de un tipo de energía a otra... Cuando hablamos del roce ¿qué pasa?... el roce produce desgaste..., el efecto del roce en la energía es igual; no es como el efecto del peso; cuando un cuerpo cae desde una altura h, por ejemplo...". En él podemos ver cómo la profesora en su intento de explicar, y que los alumnos comprendan el efecto del roce en la energía, establece una analogía (que a su vez parte de una actividad previa como la 'imaginación de una situación' en la que el grupo 'visualice' el efecto del roce: 'el desgaste de'), que equipara el efecto de desgaste al que produce en la energía, incorporando inmediatamente después otra analogía, que ahora busca que los alumnos 'vean' que el efecto del peso en la energía no es de desgaste; no es 'como' el del peso. Esta última se apoya en los conocimientos previos de los alumnos, ya que antes habían

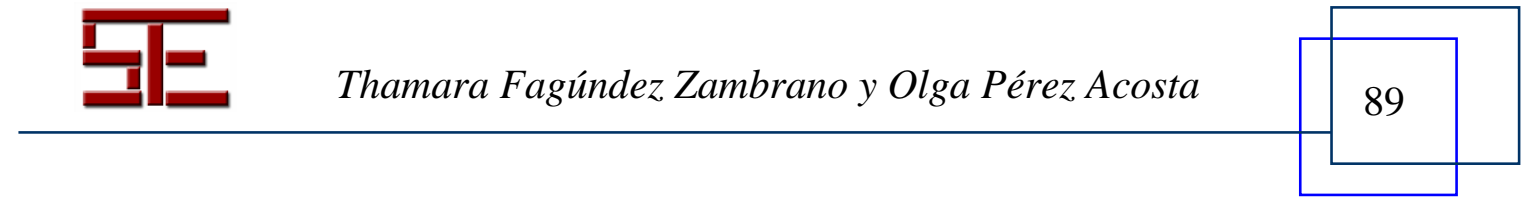




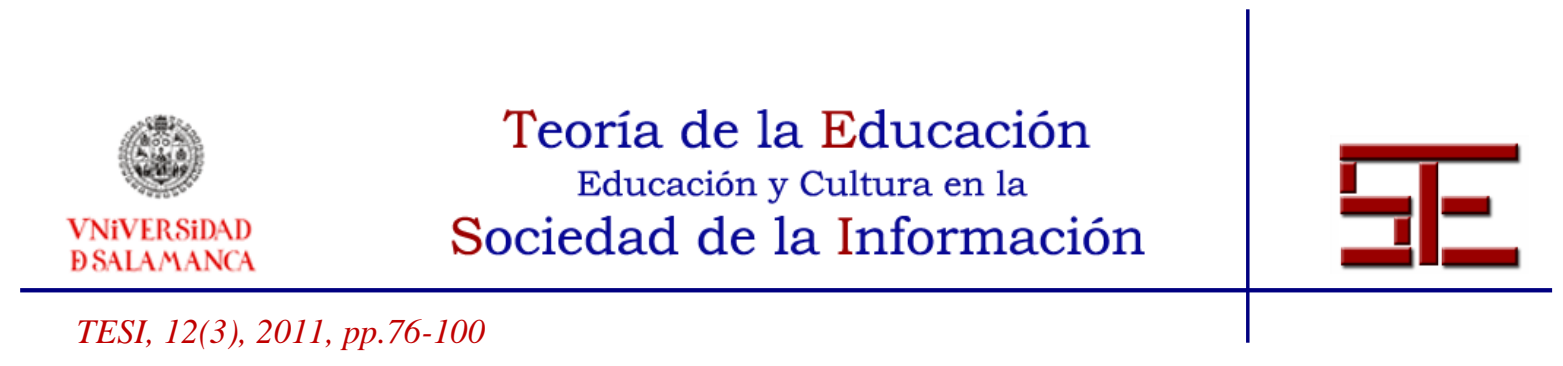

estudiado lo correspondiente a las 'fuerzas conservativas', y entre ellas conocieron que el peso es una fuerza que permite la conservación de la energía.

\subsection{Analogías para establecer y/o referenciar un marco teórico de análisis}

En este fragmento la profesora B desea que los alumnos analicen una situación planteada en base a la relación existente entre la velocidad constante con la que se desplaza un cuerpo cualquiera y la potencia desarrollada por éste tomando como referencia las leyes de Newton. Para esto, establece una analogía, que, a nuestro criterio, no presenta de forma clara la relación entre lo que se quiere estudiar y la comparación misma.

Profesora: ...Un cuerpo a velocidad constante... mientras más rápido sea esa velocidad mayor será su potencia ¿cierto o falso?... Un carro se mueve a velocidad constante de $20 \mathrm{~m} / \mathrm{s}$ y otro carro se mueve a velocidad constante de $40 \mathrm{~m} / \mathrm{s}$....el que se mueve más rápido es porque (tiene mayor potencia) $[\ldots]$

Alumno 1: Cierto.

Profesora: ¿Cierto?, ¿por qué?

Alumno 1: Si tiene mayor velocidad es porque tiene más potencia.

Profesora: Por aquí me dicen que si tiene mayor velocidad es porque tiene más potencia. Por allá [señala a un alumno al fondo del aula] ¿qué dicen?

Alumno 2: Es falso.

Profesora: dicen que es falso, ¿por qué?

Alumno 2: Bueno, ¿no es que depende de las condiciones del sistema?

Profesora. Okey, sí. Estamos en el mismo sitio; es más, es el mismo carro, solamente que uno es de tres cilindros y el otro es de cuatro cilindros. Ustedes saben que los carros los sacan así [ ] de varias cilindradas para tener más potencia [ ]. ¿Qué hacen los cilindros en el carro?

Alumno 3: Inaudible.

Profesora: Uhhh [...] no es qué hacen en sí, es como si yo tuviera cuatro obreros para cargar mil sacos o en vez de cuatro obreros pongo ocho. Entonces ocho obreros cargando los mismos mil sacos, obviamente lo van a hacer más rápido, y es mejor. Ahí lo mismo pasa con la cilindrada. El aumento de la cilindrada optimiza el trabajo del motor, puede hacer más trabajo en menos tiempo, es decir más potente es. Okey. Repito, el mismo carro, las mismas condiciones, lo único es que uno es de tres y el otro es de cuatro cilindros, uno va a $20 \mathrm{~m} / \mathrm{s}$ de aquí a allá [hace gesto indicando esto] el otro a $40 \mathrm{~m} / \mathrm{s}$ también de aquí a allá [repite gesto anterior, indicando esto]. Yo puedo afirmar que el carro que va más rápido desarrolla mayor potencia ¿sí o no? [...].

Alumnos. Cierto.

Profesora: Cierto. ¿Todos estamos de acuerdo? [mira al grupo].

Alumnos: Sííí.

Alumno 4: Profesora, es falso porque independientemente de que tenga más cilindros si se mueve a velocidad constante no hay fuerza aplicada.

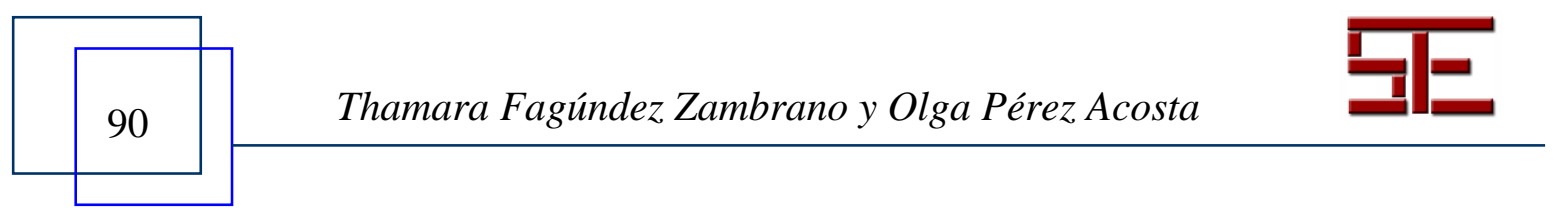




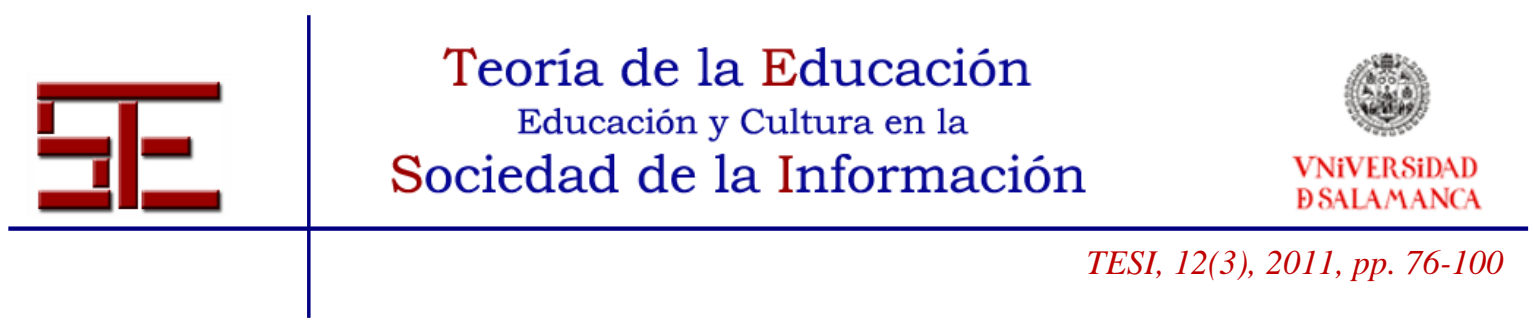

Profesora: (Esa es una explicación [...] es falso, ¿por qué?) porque si la velocidad es constante no hay aceleración no hay fuerza aplicada, no hay trabajo, no hay potencia...

La analogía se establece específicamente entre el efecto del incremento de la 'cilindrada' de los coches y el incremento del número de personas para realizar una misma tarea; al final establece que: "Los ocho obreros cargando los mismos mil sacos, obviamente lo van a hacer más rápido, y es mejor...lo mismo pasa con la cilindrada. El aumento de la cilindrada optimiza el trabajo del motor, puede hacer más trabajo en menos tiempo".

En este caso, no observamos relación alguna entre la analogía incorporada a la explicación, y el aspecto que se venía discutiendo, y que tenía que ver con la determinación de la relación entre la velocidad de un cuerpo y la potencia que éste podía desarrollar. Percibimos el intento de la profesora por usar una analogía como estrategia para la construcción de significados; pero que en este caso, consideramos, resultó inadecuada. Observamos que la situación puede ser perfectamente analizada considerando sólo el hecho de que la velocidad de los coches involucrados es constante (Primera Ley de Newton). La cuestión de la utilización de analogías confusas y poco clarificadoras puede contribuir a que la comparación no surta el efecto deseado o que incluso llegue a inducir errores conceptuales; por tanto, representa un aspecto que los profesores debemos tener en cuenta a la hora de elaborarlas e incorporarlas en nuestras explicaciones.

\subsection{Analogías como referencia o guía para establecer una metodología y/o procedimiento}

En un fragmento diferente, encontramos una situación en la que la profesora $\mathrm{C}$ intenta la elaboración de la entidad energía potencial elástica. A este nivel del curso, ya los alumnos han pasado por el proceso de construcción de significados referidos a la entidad energía potencial gravitatoria; su definición, expresión matemática, cálculo e interpretación. Observamos en el episodio el hacer de la profesora para que el grupo visualice la diferencia en cuanto al procedimiento para fijar la posición de equilibrio (X $=0$ ) de un resorte fijo por un extremo a una pared y por el otro a un bloque de masa $\mathrm{m}$ que puede desplazarse sobre una superficie horizontal. El fragmento del episodio lo mostramos a continuación:

Profesora:... Tenemos entonces la posición desde (la posición no deformada) - aquí también lo llamamos origen - y en el caso de la energía potencial gravitatoria lo llamamos nivel de referencia. Importante ahora (no-pue-do es-co-ger) [mueve la mano izquierda repetidamente] el

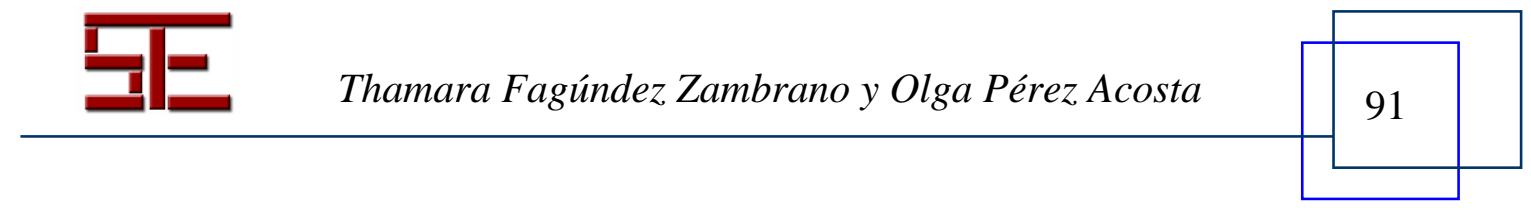




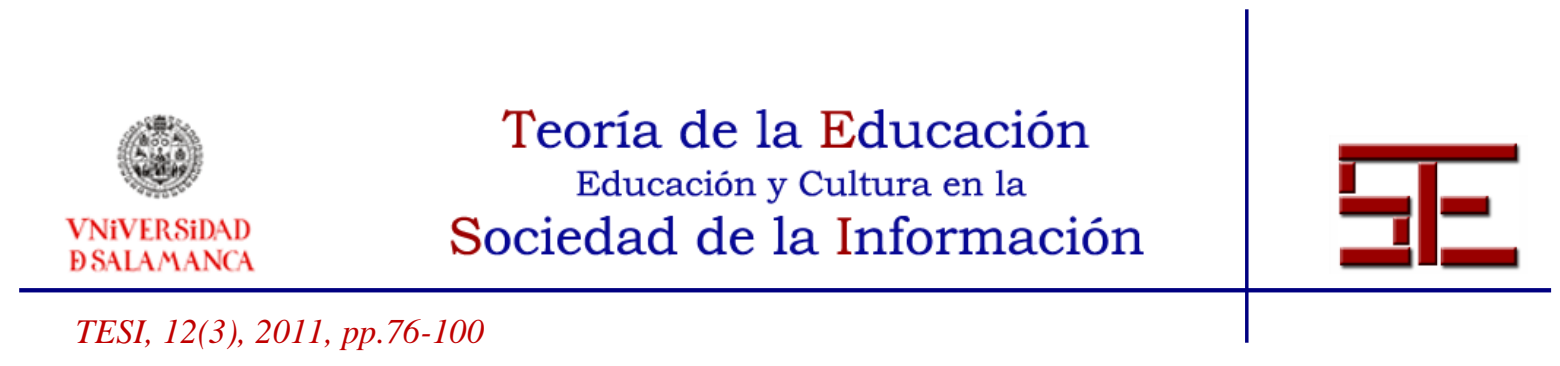

origen o nivel de referencia que yo quiera, eso era por allá en energía potencial gravitatoria; pero en el caso de la potencial elástica no podemos hacer eso; en el caso de la energía potencial elástica debo colocar el origen en la posición (no deformada) del resorte; y cualquier posición de la partícula respecto al origen será siempre tomada desde la posición no deformada; no podemos tomarla desde otro punto. Cuando yo comprimo o estiro el resorte [realiza gestos de estirar y comprimir en los dibujos antes realizados] ¿qué pasa cuando lo suelto?, el bloque comienza un movimiento. ¿hacia dónde?

Alumnos: Hacia la posición no deformada.

Profesora: [Asiente con la cabeza] La fuerza que ejerce el resorte sobre el bloque se expresa por la ley de Hooke, y ésta, sabemos que dice que la fuerza elástica es K por X, donde X es la posición del cuerpo respecto a la posición de no deformación o punto de equilibrio. Entonces cuando el cuerpo está en la posición de equilibrio, la fuerza elástica ¿vale?

Alumnos: Cero.

Profesora: Vale cero porque el cuerpo se encuentra (justo) en la posición de no deformación. O sea que la fuerza asociada a la energía potencial elástica, la fuerza elástica, vale cero cuando el resorte no está deformado.Y -ustedes ya saben- cuando hablamos de la energía potencial gravitatoria que el nivel de referencia se ubicó tomando en cuenta lo que nos dice la teoría, que es que tal nivel se ubica en el punto en el cual la fuerza asociada a (esa energía potencial) se anula, es cero. También, como nos dice la teoría asignamos (arbitrariamente) el valor de cero a la energía potencial gravitatoria en ese punto. Igual hacemos con la energía potencial elástica. Vemos que la fuerza asociada a esta energía es la elástica, y que se hace cero en la posición donde el resorte no está deformado, por tanto, en ese punto fijaremos nuestro nivel de referencia u origen.

En la energía potencial gravitatoria, vimos que el (nivel cero de energía potencial) se encontraba en el centro de la Tierra, que es el punto donde la fuerza gravitatoria se anula; pero también vimos que los movimientos de los cuerpos que estudiamos en nuestro mundo macroscópico no llegan al centro terrestre, por tanto y (por conveniencia) decidimos fijarlo, en términos generales, donde quisiéramos - ya sabemos que este quisiéramos es relativo y que dependerá de aspectos como el evitarnos trabajar con energías potenciales negativas- (pero) en el caso de la (energía potencial elástica), repito (no podemos escoger el nivel de referencia), no tenemos necesidad de hacerlo. Siempre será o coincidirá con la posición de no deformación del resorte - y que es el mismo donde la fuerza elástica es cero...

La analogía se establece confrontando la forma de fijar el 'nivel de referencia' para el caso de la energía potencial gravitatoria, haciendo hincapié en la posibilidad de fijar o 'escoger' dicho nivel, a diferencia del caso del resorte, en el que siempre el punto de equilibrio o nivel de referencia 'debe' coincidir con la posición de no deformación del mismo. Observamos que la profesora se apoya, desde el inicio del fragmento mostrado, en los conocimientos previos del grupo clase respecto a la entidad energía potencial gravitatoria. A lo largo del mismo encontramos continuas referencias a los conocimientos construidos respecto a tal entidad, y aunque no encontremos, sino sólo en una ocasión, explícitamente una analogía estructurada en términos de nexos conjuntivos, de igual manera consideramos que existen en el fragmento más de una

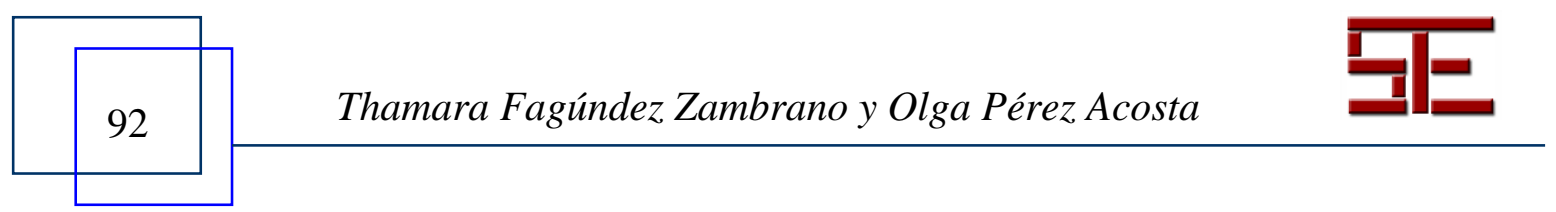




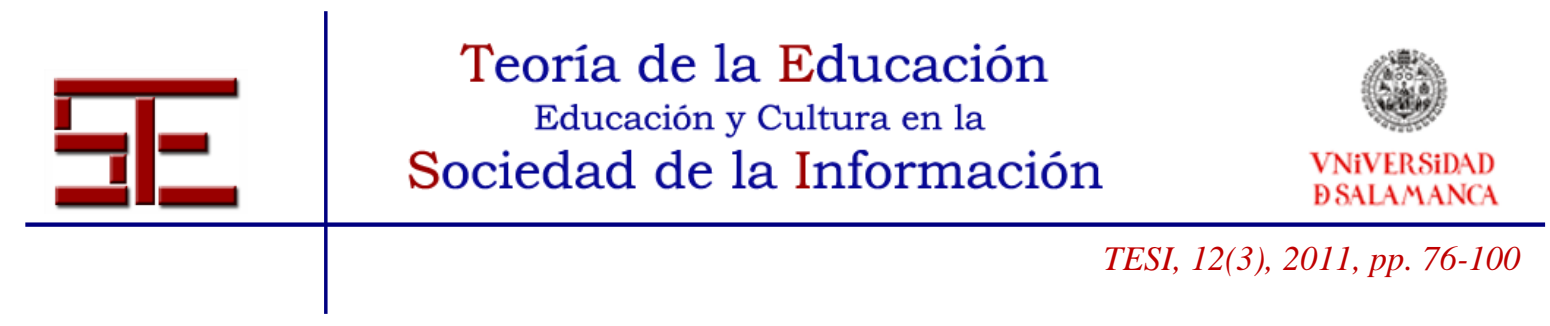

sola. Del pasaje podemos extraer tres segmentos en los que consideramos que la profesora está apoyándose en el uso de la analogía:

Analogía a:

Ahora (no-pue-do es-co-ger) [mueve la mano izquierda repetidamente] el origen o nivel de referencia que yo quiera, eso era por allá en energía potencial gravitatoria; pero en el caso de la potencial elástica no podemos hacer eso...

Interpretamos que tal enunciado equivale a decir: la escogencia del nivel de referencia para la energía potencial elástica no es como se hacía para la energía potencial gravitatoria.

\title{
Analogía b:
}

...Ustedes ya saben- cuando hablamos de la energía potencial gravitatoria el nivel de referencia se ubicó tomando en cuenta lo que nos dice la teoría, que es que tal nivel se ubica en el punto en el cual la fuerza asociada a (esa energía potencial) se anula, es cero. También, como nos dice la teoría asignamos (arbitrariamente) el valor de cero a la energía potencial gravitatoria en ese punto. Igual hacemos con la energía potencial elástica. Vemos que la fuerza asociada a esta energía es la elástica, y que se hace cero en la posición donde el resorte no está, deformado, por tanto, en ese punto fijaremos nuestro nivel de referencia u origen...

En este segmento observamos de forma más directa la analogía llevada a cabo; cuando identificamos la conexión entre un aspecto que tiene que ver con el procedimiento en general para fijar el nivel de referencia correspondiente a la energía potencial con el procedimiento a seguir para fijar el nivel de la energía potencial elástica. Tal conexión comparativa la notamos por la incorporación de la construcción 'igual hacemos'.

\section{Analogía c:}

\begin{abstract}
En la energía potencial gravitatoria, vimos que el (nivel cero de energía potencial) se encontraba en el centro de la Tierra, que es el punto donde la fuerza gravitatoria se anula; pero también vimos que los movimientos de los cuerpos que estudiamos en nuestro mundo macroscópico no llegan al centro terrestre, por tanto y (por conveniencia) decidimos fijarlo, en términos generales, donde quisiéramos- ya sabemos que este quisiéramos es relativo y que dependerá de aspectos como el evitarnos trabajar con energías potenciales negativas- (pero) en el caso de la (energía potencial elástica), repito (no podemos escoger el nivel de referencia), no tenemos necesidad de hacerlo. Siempre será o coincidirá con la posición de no deformación del resorte - y que es el mismo donde la fuerza elástica es cero...
\end{abstract}

En este segmento también de forma 'tácita' vemos que se hace una analogía, que pudiera expresarse en pocas palabras de la manera siguiente: "El nivel de referencia para la energía potencial elástica se fija siempre en la posición de no deformación del

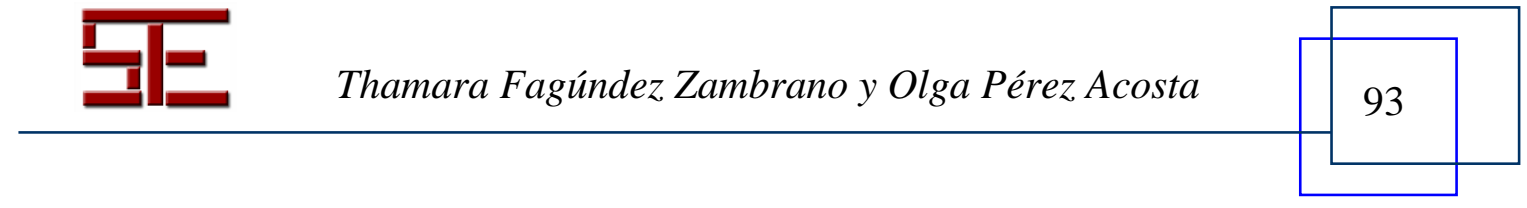




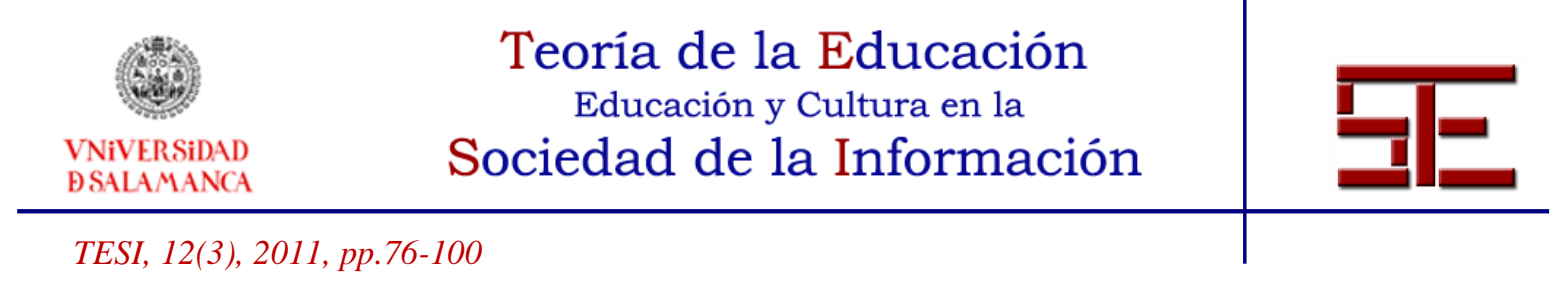

resorte, y no como en el caso de la energía potencial gravitatoria en la que lo fijamos según nuestra conveniencia". Destacamos que tanto en éste como en el primer segmento, la comparación se hace, desde el punto de vista retórico argumentativo (Perelman ; Olbrechts-Tyteca, 1989), por oposición.

\section{6.- CONCLUSIONES}

6.1 Respecto al objetivo: Analizar cómo los profesores de física de nivel universitario utilizan las analogías para construir explicaciones y argumentaciones

En base a nuestros análisis detectamos que las profesoras incorporan en sus explicaciones una variedad de recursos argumentativos que contribuyen a una construcción de conocimientos menos memorística y más significativa, por su enorme potencial para contribuir al aprendizaje a partir de lo ya conocido y para relacionar y organizar el nuevo conocimiento; para transformarlo y/o para sugerir nuevos significados al mundo material. La forma de trabajo en las clases nos afianzar en la visión de unas profesoras que pretenden una enseñanza no 'impositiva' sino 'razonada' y/o argumentada.

La analogía es uno de los recursos más usados por las profesoras para aportar a los alumnos razones 'convincentes', trasladando los significados cotidianos compartidos a científicos que habrá que compartir mediante el establecimiento de relaciones entre los mismos. Las situaciones de aprendizaje o de construcción de conocimientos planteadas usando a la analogía como recurso se estructuran intentando impulsar a los alumnos a recurrir a sus conocimientos previos, intereses y experiencias, apelando a hechos cotidianos, a aplicaciones de los contenidos a situaciones reales y al establecimiento de relaciones con contenidos de otras asignaturas. Aun así, según nuestro punto de vista, en las explicaciones estudiadas echamos en falta una mayor presencia de situaciones contextualizadas y una indagación más profunda sobre los conocimientos previos e intereses de los alumnos.

Resultó ser el recurso usado con preferencia por las tres profesoras para introducir diferentes temas de las últimas unidades de la asignatura y que comprenden el estudio del movimiento de rotación pura (cinemática y dinámica).

6.2.-Respecto al objetivo: Identificar las analogías presentes en las explicaciones elaboradas en clases universitarias por profesores experimentados de física en el contexto de una facultad de ingeniería.

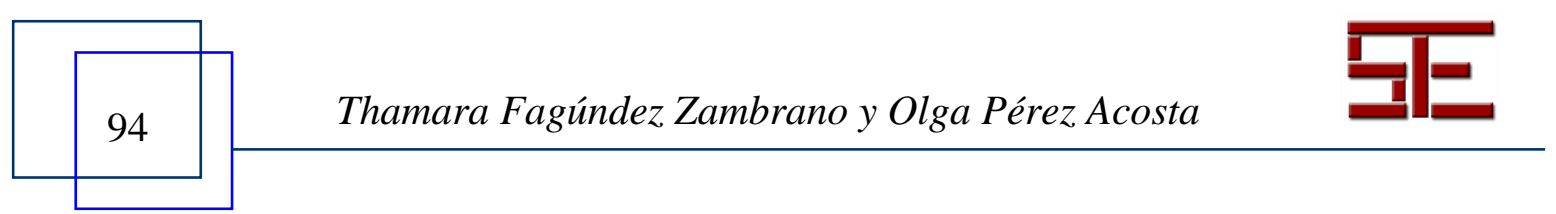




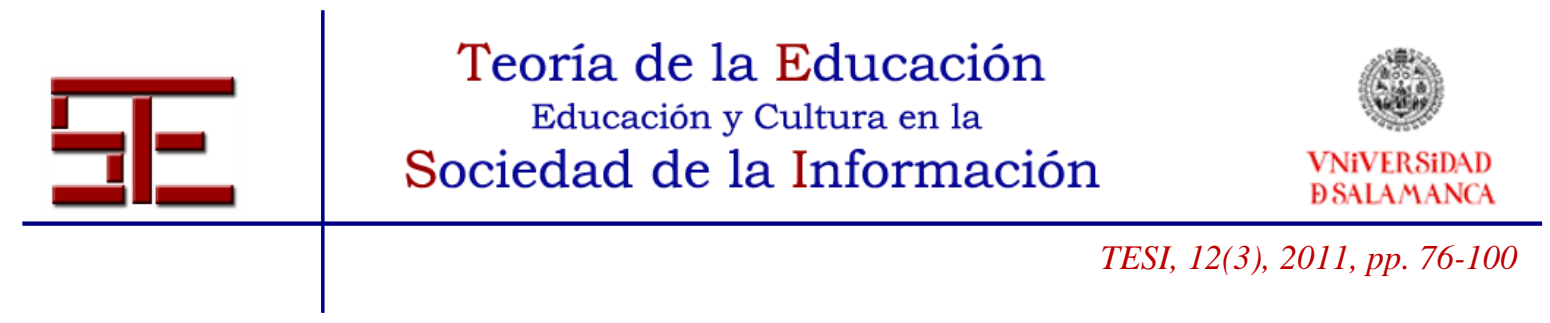

En las analogías elaboradas se 'comparan' entidades de campos científicos diferentes. Otras veces, la comparación se hace entre entidades del campo científico y cotidiano. El uso de estos recursos lo observamos en las tres profesoras, y con marcada preferencia, a partir del segundo tercio de la asignatura, y para introducir los temas que tienen que ver con el movimiento de rotación pura; tanto en la parte cinemática como en la dinámica. Identificamos que en términos generales en las clases de física analizadas las analogías son usadas para:

a. La construcción de significados relacionados con conceptos científicos abstractos

b. Establecer el comportamiento de entidades y/o sistemas físicos

c. Establecer y/o referenciar un marco teórico de análisis

d. Como referencia para establecer una metodología y/o procedimiento

Encontramos, en diferentes episodios analizados, el planteamiento de analogías de forma 'tácita'; es decir, no de la manera tradicional como ésta se concibe, 'A es como $\mathrm{B}$ '. Este aspecto resultó ser repetitivo en las tres profesoras cuyas explicaciones fueron objeto de nuestro estudio.

6.3.- Respecto al objetivo: Identificar y analizar la contribución de las analogías en las explicaciones para la construcción de significados científicos

Consideramos que el objetivo de la incorporación de las analogías en las clases de física analizadas es ayudar a los alumnos a comprender o clarificar el concepto, acercar el concepto o entidad a algo más familiar para ellos. Permitir el acercamiento del alumno a ideas que pueden resultar ser muy alejadas de su contexto cotidiano, convertir un concepto abstracto en algo más concreto, ayudando a visualizar la entidad y contribuyendo a fomentar la capacidad de abstracción. Por su naturaleza actúan como 'puentes' que relacionan y, a la vez, facilitan la conexión del conocimiento, científico o social, previo de los alumnos y el nuevo conocimiento a aprender.

Al servir para comparar, evidenciar, representar y explicar algún objeto, fenómeno o situación (al igual que las comparaciones y metáforas), tienen un enorme potencial para contribuir al aprendizaje a partir de lo ya conocido y para relacionar y organizar el nuevo conocimiento; para transformarlo y/o para sugerir nuevos significados al mundo material. En la mayoría de los casos la utilización de estos recursos obedece, como en la vida cotidiana, a la espontaneidad; en general, en el contexto de la enseñanza, no hay una aplicación conscientemente planificada de los mismos como recursos valiosos para aprender.

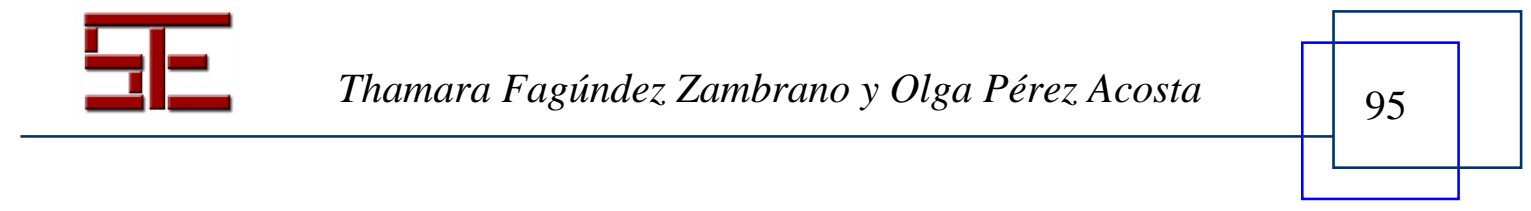




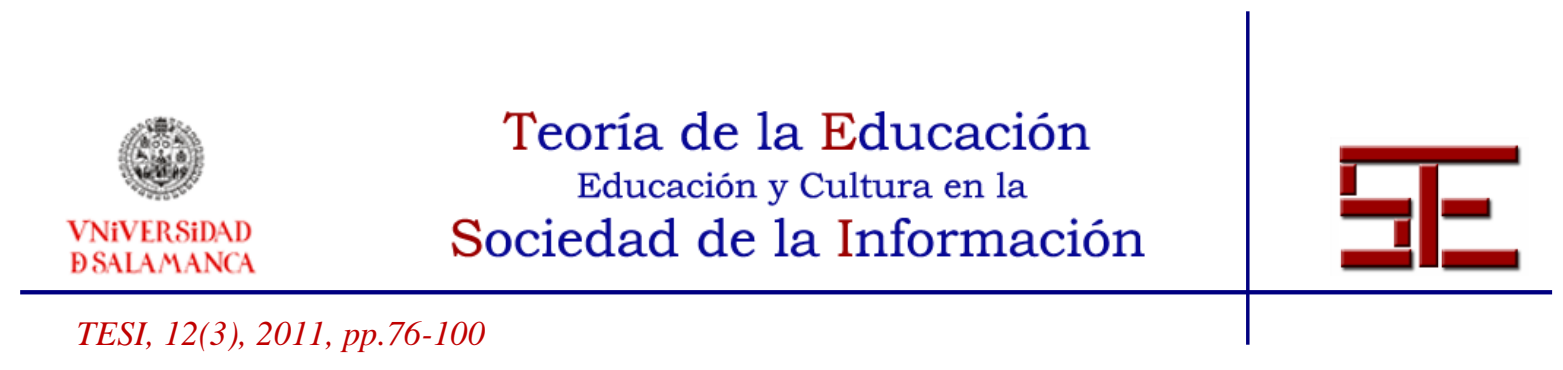

Un aspecto que hay que tener en cuenta es que la elaboración de analogías confusas y poco clarificadoras puede contribuir a que la explicación no surta el efecto deseado: contribuir a la construcción de significados, e incluso, llegar a inducir a los alumnos a 'afianzar' concepciones alternativas sobre algunos aspectos de la física, si las tuvieren.

Detectamos que algunas de las analogías establecidas (por ejemplo, las analogías entre las cantidades o magnitudes físicas lineales con la del movimiento circular o de rotación) no cumplen con las exigencias de analogía usada para enseñar y aprender; por tanto consideramos que éstas no son las más adecuadas ya que son equivalencias, el análogo, en tal caso, es la forma de definir algunas de las variables del movimiento de rotación. Las analogías, en ciencia, establecen relaciones entre dos conceptos o problemas diferentes, tanto similitudes y cómo diferencias, evitando asociar al concepto el análogo.

Por otra parte, consideramos que, al estudiar el concepto de campo gravitatorio, la analogía con el aura y con los campos eléctricos y magnéticos confunden al estudiante, en primer lugar, porque no hay una visualización de conceptos abstractos (campo) con algún fenómeno inobservable, en segundo lugar, no hay una Implicancia cognitiva y afectiva de los alumnos que favorezca la disposición por aprender. Se recomienda estudiar el concepto de campo, con alguna analogía concreta donde los alumnos puedan observar, sentir, oler, etc, y luego extender a los otros campos como son eléctricos y magnéticos.

Participamos de la consideración sobre la riqueza y pertinencia de los recursos elaborados en base a experiencias de la vida cotidiana para el estudio y aprendizaje de las ciencias, y, por tanto, para la construcción de significados especializados, por su contribución a mostrar la ciencia no demasiada alejada de situaciones conocidas por los alumnos (Lemke, 1997). Desde tal punto de vista, en las lecciones analizadas se encuentra a faltar una mayor presencia de explicaciones que incluyan situaciones en que los alumnos logren establecer relaciones entre los conocimientos o entidades científicas que se construyen y su contexto cotidiano. De igual forma, creemos en la conveniencia de presentar más 'contextualizaciones', basadas en situaciones y/o experiencias que relacionen los nuevos conocimientos con el quehacer del profesional de la ingeniería, la industria y la tecnología; así como mediante el establecimiento de la 'utilidad' de tales conocimientos y sus aplicaciones en tales campos y en la vida cotidiana.

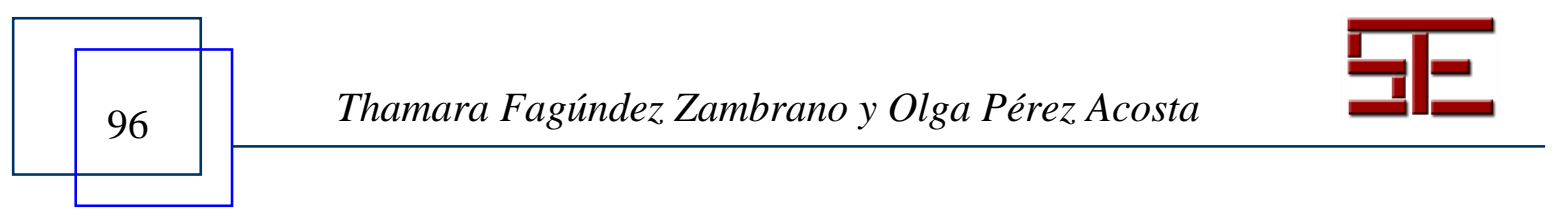




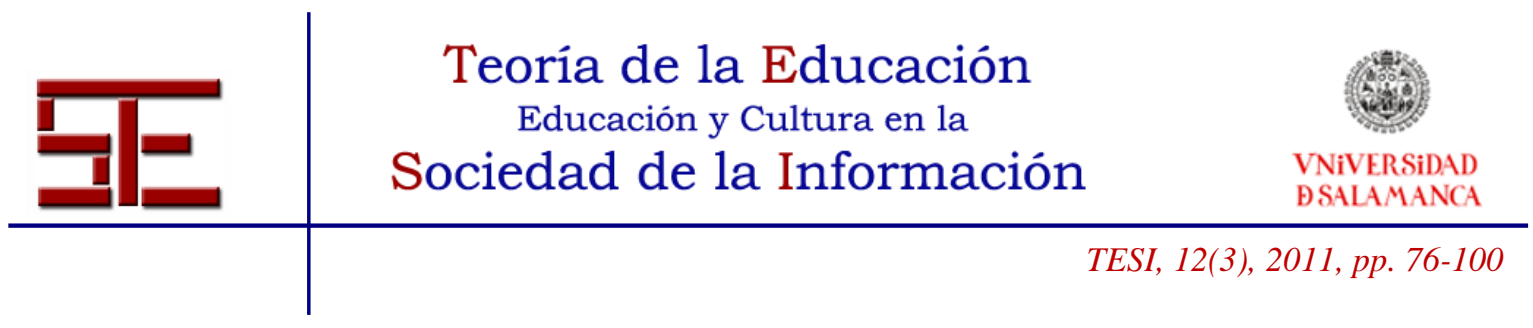

\section{7.- IMPLICACIONES PARA LA MEJORA DE LA PRÁCTICA DOCENTE}

Consideramos que el razonamiento analógico puede y debe tener un papel relevante dentro de los procesos de aprendizaje de las Ciencias en general, no sólo a través de la construcción y uso de analogías funcionales o comunicativas, sino también a través de analogías explicativas que sirvan para ayudar a los alumnos a relacionar nociones que para ellos están muy distantes pero que, desde el punto de vista científico, tienen una misma explicación. La posibilidad de emplear la enseñanza basada en analogías reúne una serie de ventajas que responden a una visión constructivista del aprendizaje.

En general, puede considerarse que una analogía adecuada permite establecer relaciones entre los nuevos conceptos con lo que el estudiante ya conoce; son las que inducen a los estudiantes a conectar conceptos relacionados y promueven la formación de sistemas conceptuales; y una analogía no adecuada es aquella en la que es difícil identificar y 'mapear' las características relevantes compartidas por el análogo y el blanco. Por otra parte, el uso de analogías en la enseñanza de las ciencias de manera pasiva, sin analizar su papel, naturaleza, limitaciones y fortalezas, pudiera conducir al estudiante a la percepción de las analogías como el centro de lo que debe aprender y no como una herramienta para la comprensión. Tal cuestión la consideramos importante, siendo el proceso de construcción de significados involucrado requiere de un componente importante de capacidad de abstracción y de habilidades propias del pensamiento formal, por lo que, además de la analogía presentada, se hace necesaria por parte del estudiante una toma de conciencia sobre los límites y alcances de la misma. De la habilidad del estudiante para establecer relaciones y ajustarlas en procesos cognitivos abstractos dependerá la utilidad de las analogías. La investigación que realizamos no contempló el estudio de la analogía desde el punto de vista del alumno; pero queda claro que si no se toman las medidas adecuadas existen bastantes posibilidades de que el pensamiento analógico derive en la formación de concepciones alternativas o las refuerce.

\section{REFERENCIAS}

Clement, J. \& Brown, D. (1984). Using analogical reasoning to deal with "Deep". Misconceptions in Physics. (Informe No.SE048584). Washington, D.C. EE. UU.: Fund for the Improvement of Postsecundary Education.

Dagher, Z. (1995a). Analysis of analogies used by science teachers. Journal of Research in Science Teaching, 32(3), 259-270.

- (1995b). Review of studies on the effectiveness of instructional analogies in science education. Science Education, 79 (3), 295-312.

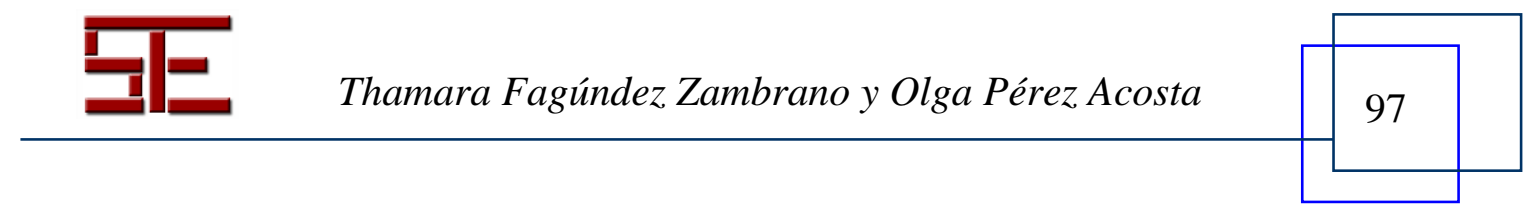




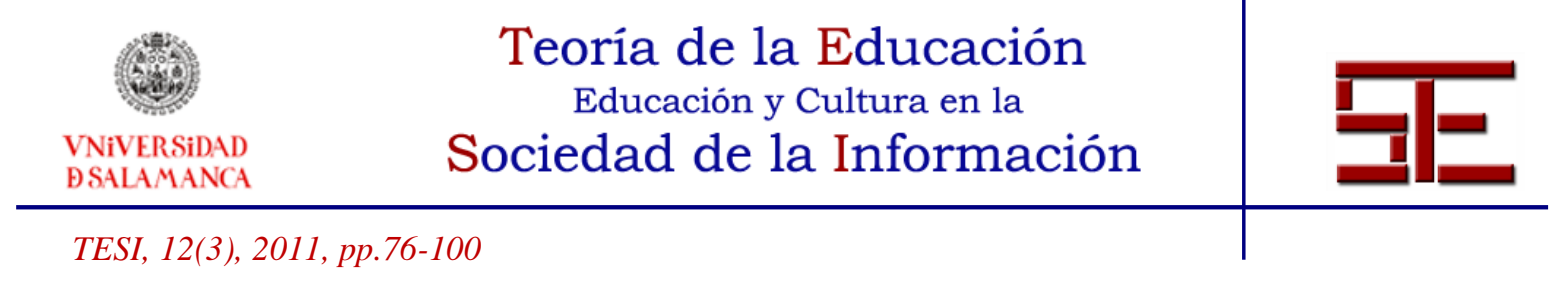

- (1992). Analysis and synthesis of studies related to the effectiveness of analogies in science learning. Paper presented at the annual meeting of the National Association for Research in Science Teaching, Boston, M. A.

Dagher, Z. \& Cossman, G. (1992). Verbal explanation given by science teachers: Their nature and implications. Journal of Research in Science Teaching, 19, 361-374.

Díaz-Barriga, F. \& Hernández, R. (2002). Estrategias docentes para un aprendizaje significativo una interpretación constructivista. $2^{\mathrm{a}}$ Edición. México: McGraw-Hill Interamericana.

Duit, R. (1991). On the role of analogies and metaphors in learning science. Science Education, 75, 649-672.

Estrada C. \& Godoy L. (2007). Análisis de analogías en la mecánica computacional y estructural. Mecánica computacional. (XXVI) ,2456-2470.

Fernández, G., González, G. \& Moreno J. (2004). Consideraciones acerca de la investigación en analogías. Estudios Fronterizos, (5) 79-105.

Glynn, S., Britton, B., Semrud-Clikman, M. \& Muth, K. (1989). Analogical Reasoning and Problem Solving in Science Textbooks. En Glover, J., Ronning, R. \& Reynolds, C. (Ed), Handbook of Creativity. (pp. 383-398) New York: Plenum Press.

Godoy, A. (2002a). Éxitos y problemas de las analogías en la enseñanza de la mecánica. Journal of Science Education, (3)11-15.

- (2002b). Sobre la estructura de las analogías en ciencias. Interciencia, 27 (8): 422-429.

- (2007). Analogías en Ciencias: ¿Facilitadoras u obstaculizadoras en la construcción de conocimientos? En Félix A. Lamas La Dialéctica Clásica: La Lógica de la Investigación, Ed. Colección Circa Humana Philosophia. Buenos Aires.

González, G. (2005). El modelo analógico como recurso didáctico en ciencias experimentales. Revista Iberoamericana de Educación. Disponible en http://www.rieoei.org/1080.htm.

Lawson, A. (1993). The Importance of Analogy: A Prelude to the Special Issue. Journal of Research in Science Teaching, 30 (10), 1213-1214.

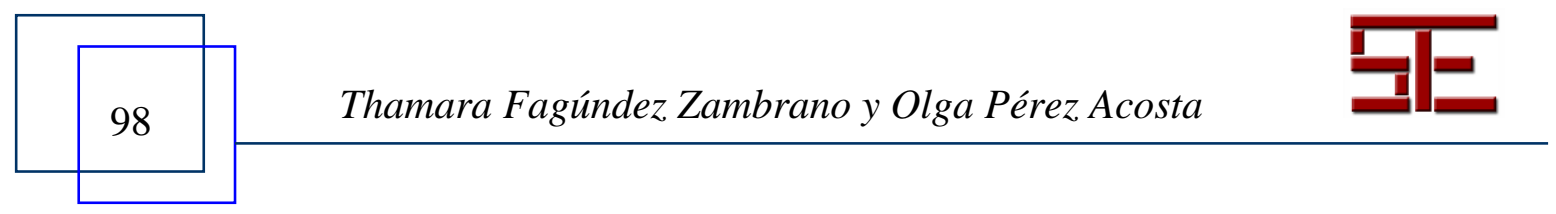




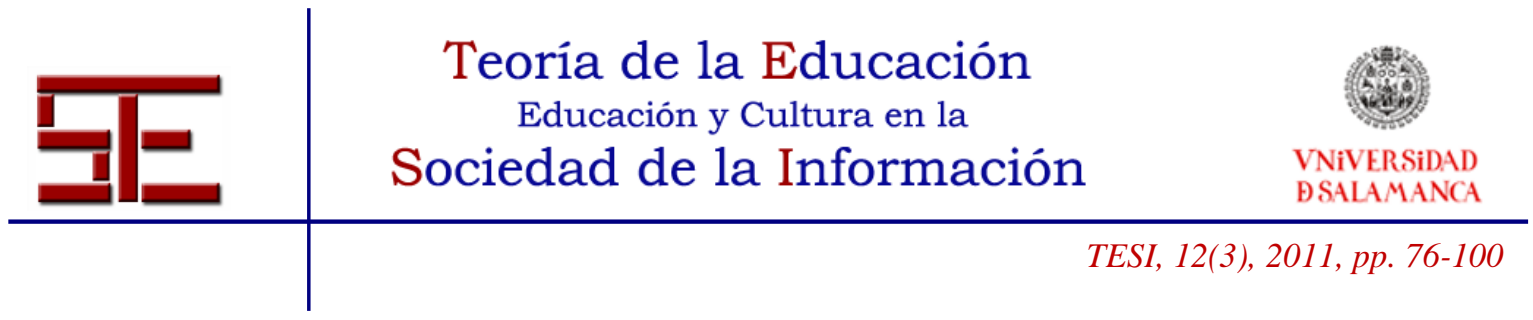

Lemke, J. (1997). Aprender a hablar ciencia. Lenguaje, aprendizaje y valores. Paidós, Barcelona, España. Trad. obra original en inglés: Talking Science: Language, learning and values, 1993).

Moro, L., Viau, J., Zamorano, H. \& Gibbs, M. (2007).Aprendizaje de los conceptos de masa, peso y gravedad. Investigación de la efectividad de un modelo analógico. Revista Eureka sobre Enseñanza y Divulgación de la Ciencia, (4), 272-286.

Ogborn, J., Kress, G., Martins, I. \& Mc Gillicuddy, K. (1996): Explaining science in the classrroom. Open University Press.

Oliva, J. (2003). Rutinas y guiones del profesorado de ciencias ante el uso de analogías como recurso en el aula. Revista Electrónica de Enseñanza de las Ciencias. (2), 1.Disponible enero 2008 en

http://www.saum.uvigo.es/reec/volumenes/volumen2/Numero1/Art2.pdf.

- (2004a). El papel del razonamiento analógico en la construcción histórica de la noción de fuerza gravitatoria y del modelo del sistema solar (Primera parte). Revista Eureka sobre Enseñanza y Divulgación de las Ciencias, 1(1). En línea en:

http://www.apaceureka.org/revista/Volumen1/Numero_1_1/Vol_1_Num_1.htm.

- $\quad$ (2004b). El papel del razonamiento analógico en la construcción histórica de la noción de fuerza gravitatoria y del modelo del sistema solar (Segunda parte). Revista Eureka sobre Enseñanza y Divulgación de las Ciencias, Vol. 1, 3, 167186. En línea en:

http://www.apaceureka.org/revista/Volumen1/Numero_1_1/Vol_1_Num_1.htm.

- (2006). Actividades para la enseñanza/aprendizaje de la química a través de analogías. Revista Eureka sobre Enseñanza y Divulgación de la Ciencia, (3),1,104-114.

Oliva, J., Aragón, M., Mateo, J. \& Bonat, M. (2001). Una propuesta didáctica basada en la investigación para el uso de analogías en la enseñanza de las ciencias. Revista Enseñanza de las Ciencias, 19 (3), 453-470.

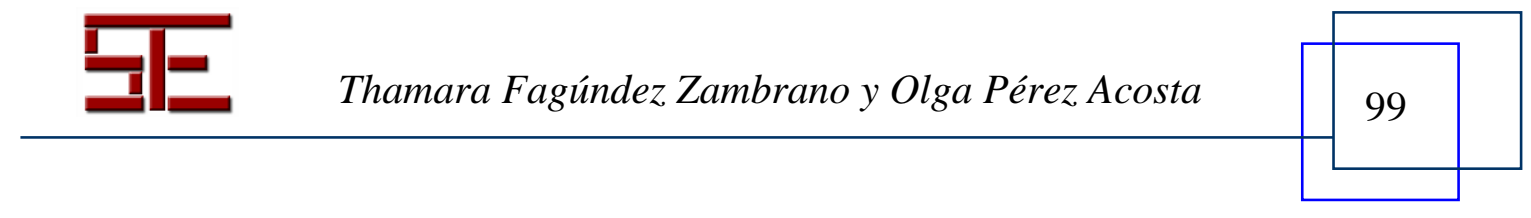




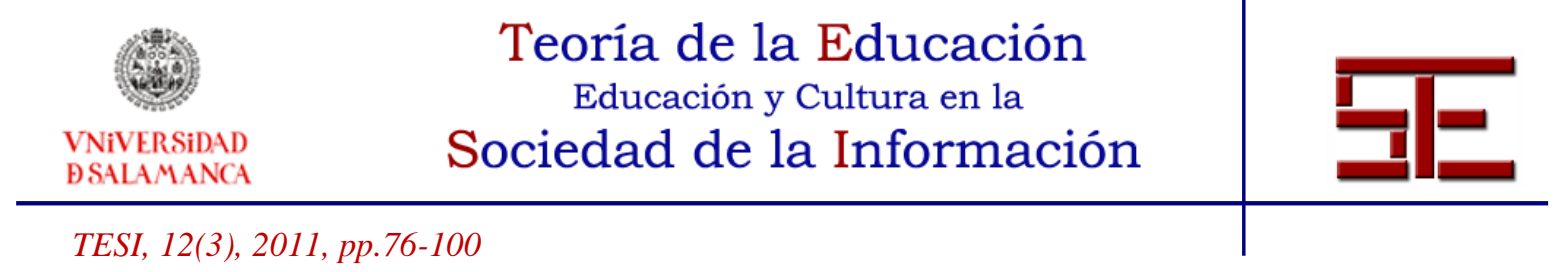

Oliva, J., \& Aragón, M. (2006). Pensamiento analógico y construcción de un modelo molecular para la materia. Revista Eureka sobre Enseñanza y Divulgación de la Ciencia. (4) 1, 21-41.

Perelman, Ch. \& Olbrechts-Tyteca, L. (1989). Tratado de la Argumentación. La Nueva retórica. Madrid: Gredos.

Podolefsky, N. \& Finkelstein, N. (2006). Use of analogy in learning physics: The role of representations. Physical Review Special Topics. Physics Education Research. Disponible en: http://prstper.aps.org/abstract/PRSTPER/v2/i2/e020101.

- (2007a). Analogical scaffolding and the learning of abstract ideas in physics:Empirical studies. Physical review special topics. Physics education research. Disponible en http://prst-per.aps.org/pdf/PRSTPER/v3/i2/e020104.

Podolefsky,N. \& Finkelstein,N.(2007b)Analogical scaffolding and the learning of abstract ideas in physics: An example from electromagnetic waves. Physical Review Special Topics. Physics Education Research. Disponible en: http://prster.aps.org/abstract/PRSTPER/v3/i1/e010109.

Stake, R. (1998). Investigación con estudio de casos. Madrid: Morata.

Treagust, D., Duit, R., Joslin, P., \& Lindauer, I. (1992). Science teachers use of analogies: Observations form classroom practice. International Journal of Science Education, 14, 413-422.

Zamorano, R., Gibbs, H. \& Viau, J. (2006). Modelización analógica en la enseñanza de circuitos de corriente continua. Journal of Science Education, (7), 30-33.

Para citar el presente artículo puede utilizar la siguiente referencia:

Fagúndez Zambrano, T. y Pérez Acosta, O. (2011). La analogía y la construcción de significados científicos en la enseñanza de la física para estudiantes de ingeniería. Revista Teoría de la Educación: Educación y Cultura en la Sociedad de la Información. 12(3), 76-100 [Fecha de consulta: $\mathrm{dd} / \mathrm{mm} / \mathrm{aaaa}$ ]. http://campus.usal.es/ revistas_trabajo/index.php/revistatesi/article/view/8484/8577

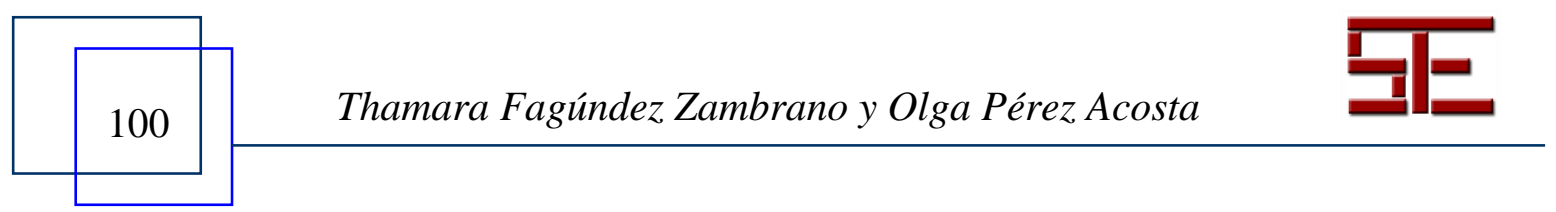

